\title{
Optical metamaterials: Possibilities and limitations
}

\author{
M. Kafesaki, R. Penciu, Th. Koschny, N. H. Shen, D. Guney, \\ J. Zhou, \\ E. N. Economou and C. M. Soukoulis
}

Foundation for Research \& Technology, Hellas (FORTH), Crete, Greece Iowa State University (ISU), USA 


\section{Optical metamaterials: Possibilities and limitations}

M. Kafesaki, R. Penciu, Th. Koschny, N. H. Shen, D. Guney, J. Zhou,

E. N. Economou and C. M. Soukoulis

Foundation for Research \& Technology, Hellas (FORTH), Crete, Greece Iowa State University (ISU), USA 


\section{Metamaterials}

Artificial, structured (in subwavelength scale) materials

Electromagnetic (EM) properties derive from shape and distribution of constituent units (artificial atoms)

EM properties not-encountered in natural materials

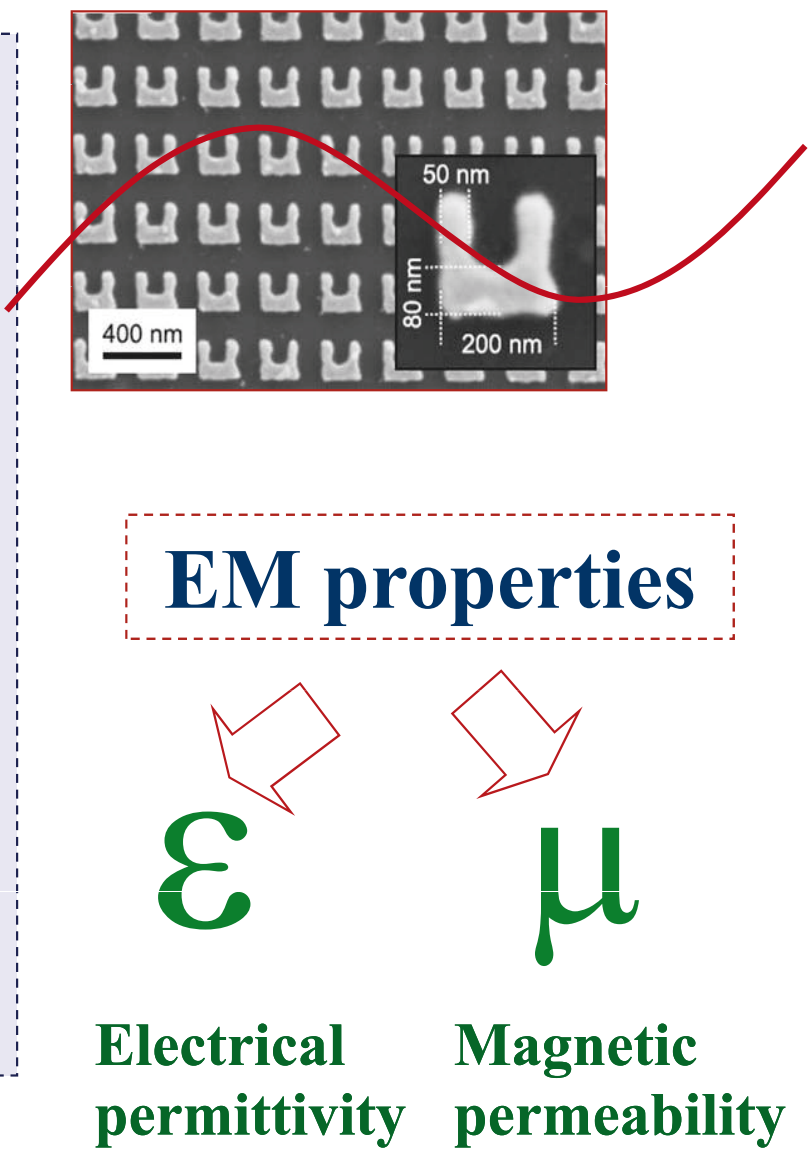

Possibility to engineer electromagnetic properties 


\section{Left-handed metamaterials}

\section{Negative electrical permittivity $(\varepsilon)$ \\ Negative magnetic permeability $(\mu)$}

Veselago (1968): How a plane wave $e^{i k x}$ propagates in media with $\varepsilon<0$ \& $\mu<0$ ?

$$
\begin{gathered}
k=\frac{\omega}{c} \sqrt{\varepsilon \mu} \\
n^{2}=\varepsilon \mu \Rightarrow n=-\sqrt{\varepsilon \mu}
\end{gathered}
$$

Negative $\varepsilon, \mu, n$

Novel and unique propagation characteristics in those materials! 


\section{Novel phenomena in left-handed materials}

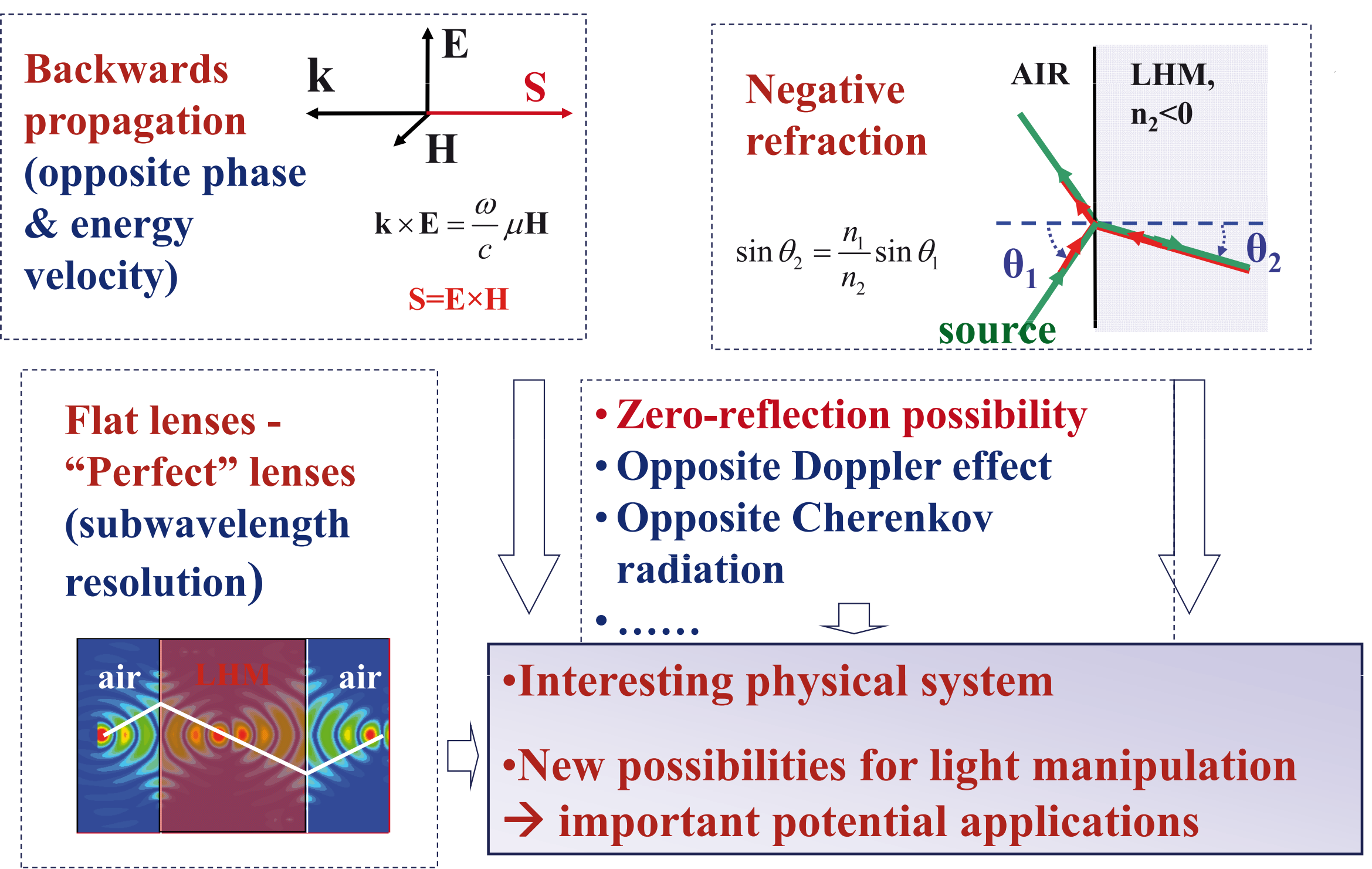




\section{Application areas of left-handed materials}

\section{New solutions and possibilities in}

-Imaging/microscopy

-Lithography

-Data storage

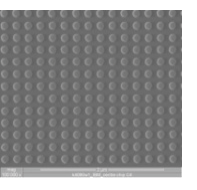

-Communications and information processing (subwavelength guides, optimized/miniaturized antennas \& filters, improved transmission lines ...)
Exploiting the subwavelength resolution capabilities of LHMs

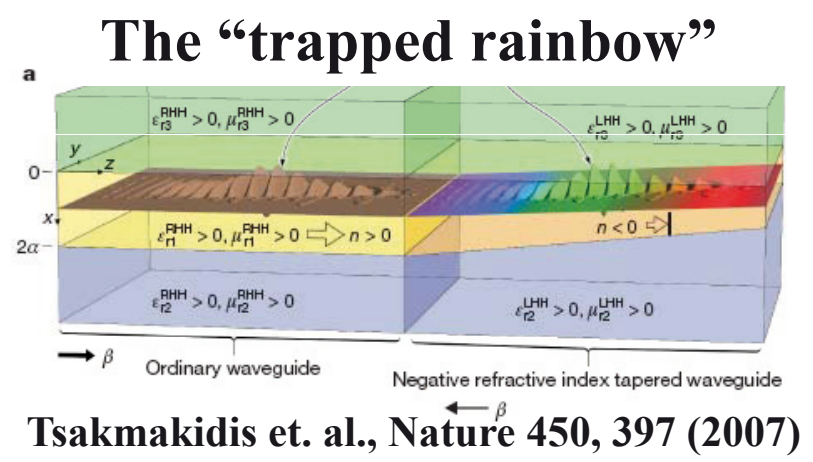

$\bullet . .$. 


\section{Metamaterials beyond negative index}

High index metamaterials

Low index metamaterials
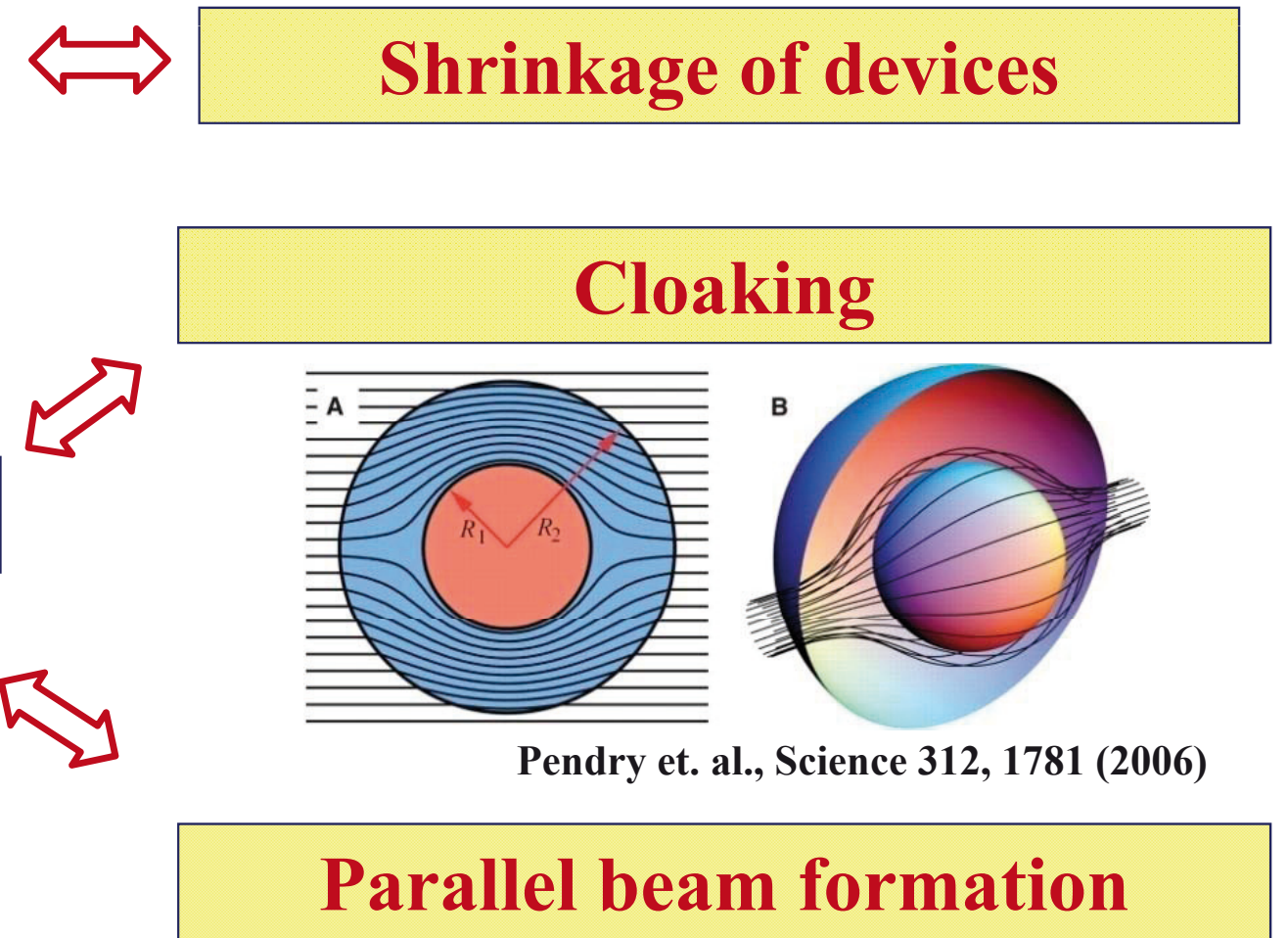


\section{"Zero"-index metamaterials}

\section{Parallel beam formation}

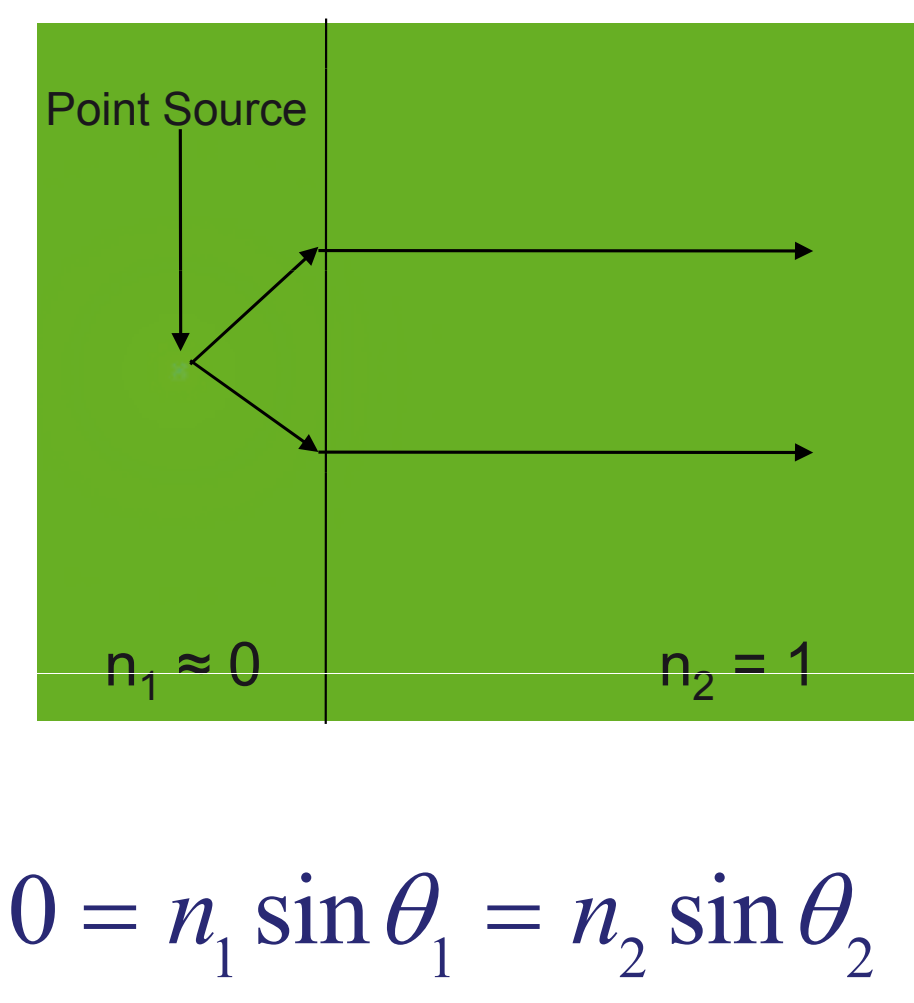

All the angles $\theta_{2}$ should be zero, and therefore perpendicular to the surface 


\section{Metamaterials beyond negative index}

High index metamaterials

Low index metamaterials
$\Leftrightarrow \quad$ Shrinkage of devices
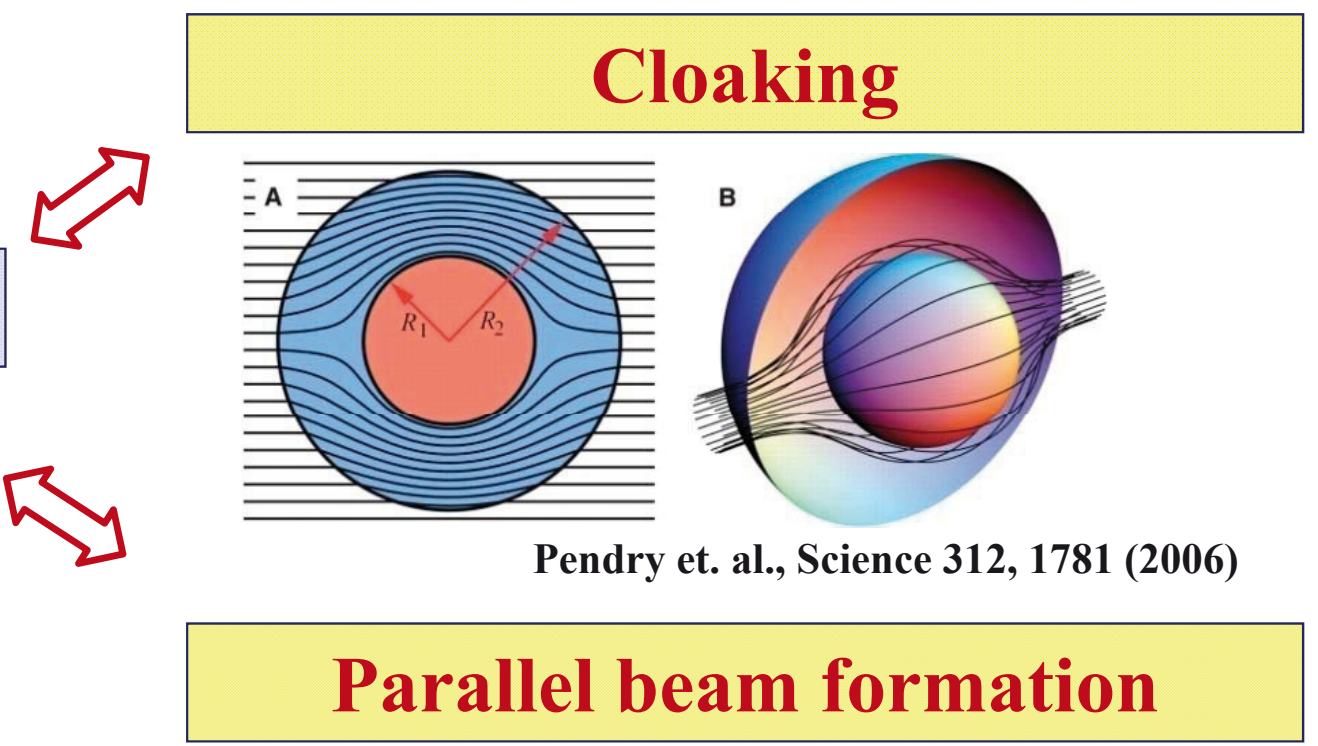

$\Leftrightarrow$

Hyperlensing

Bi-anisotropic media

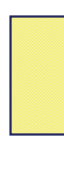

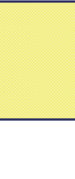

Single-negative media 


\section{Designing left-handed metamaterials}

Most common approach: Merging structures of negative permittivity $(\varepsilon)$ with structures of negative permeability $(\mu)$

Negative permeability:
Structures of resonant
loop-currents

\begin{tabular}{|l|}
\hline Negative permittivity: \\
Continuous wires
\end{tabular}

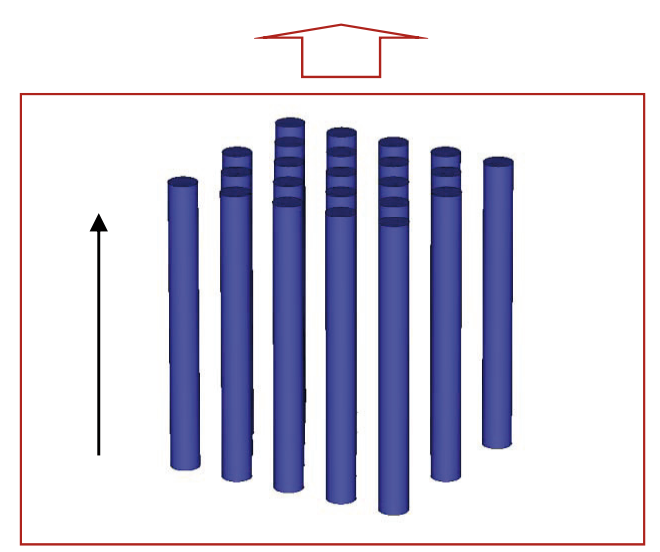

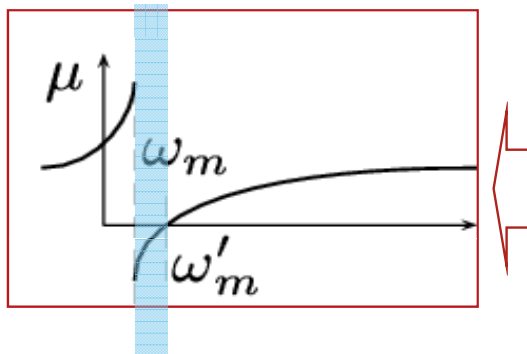
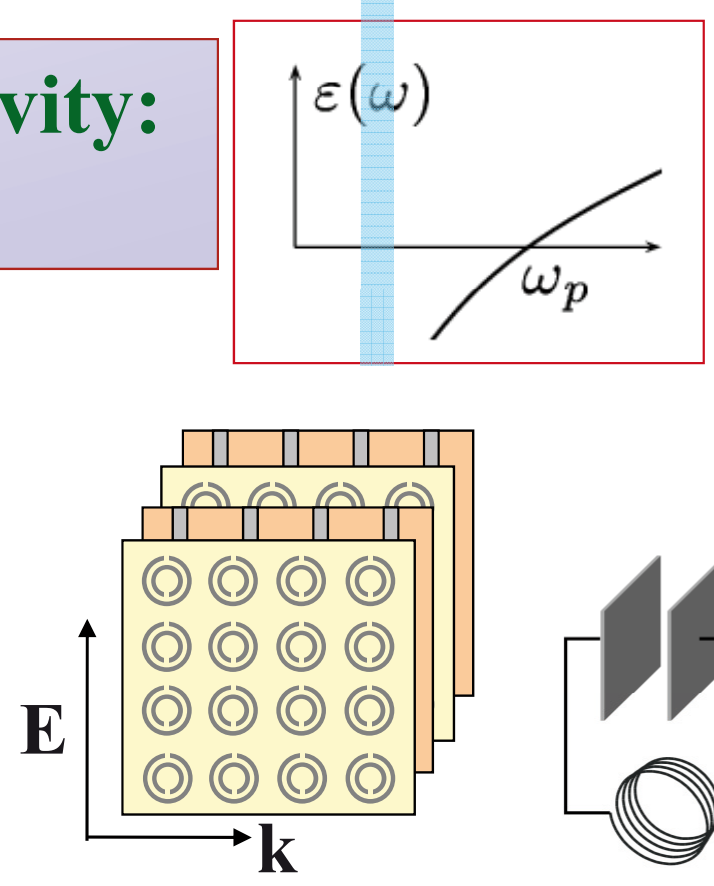

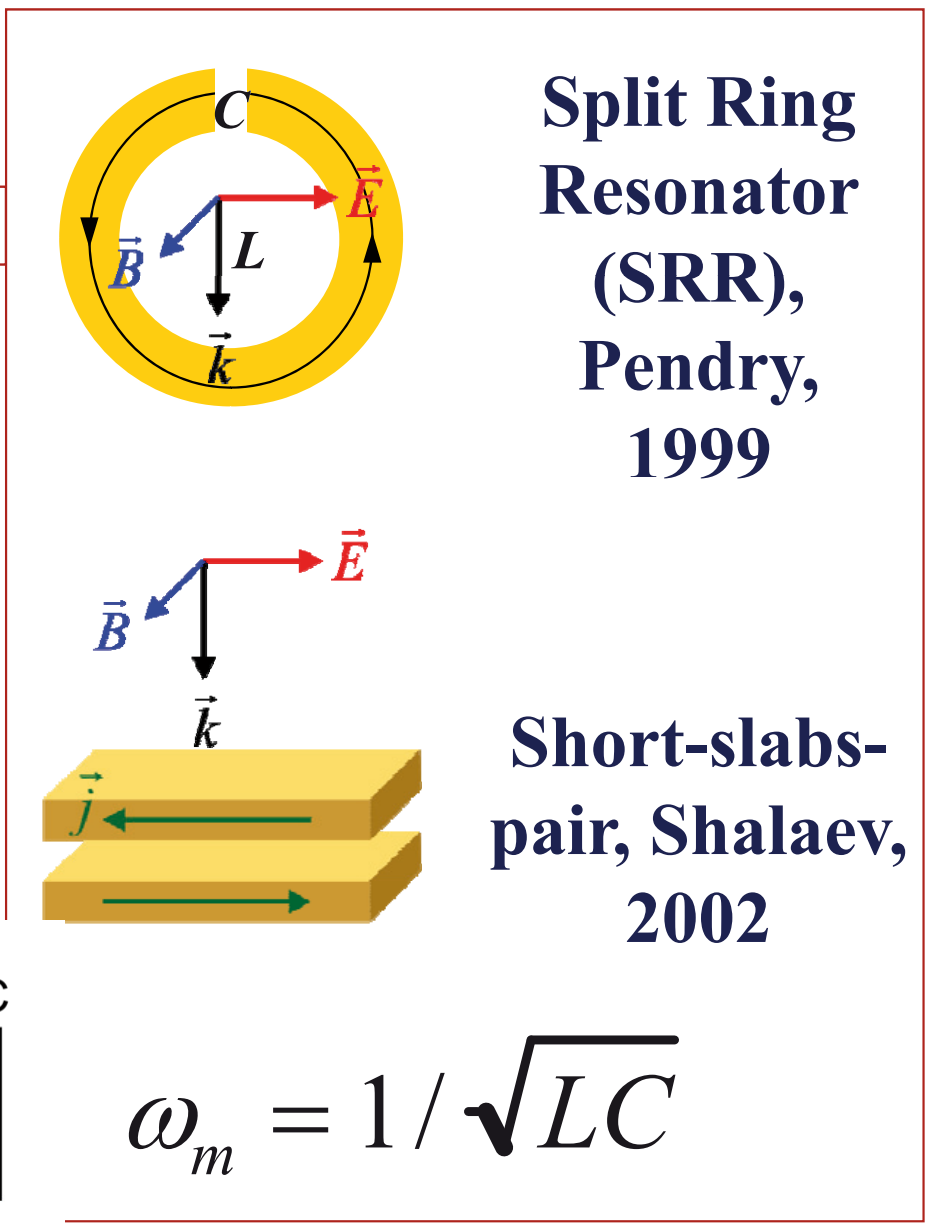




\section{Main investigation aims/directions}

- Analyze, understand, optimize and tailor metamaterial response

- Achieve optical metamaterials - reduce losses in metamaterials

- Achieve three-dimensional isotropic left-handed metamaterials

- Create switchable and tunable metamaterials

- Devise/analyze new designs and approaches for negative index behaviour (chiral or anisotropic metamaterials)

- To explore novel phenomena and possibilities in metamaterials 


\section{Main investigation aims/directions}

- Analyze, understand, optimize and tailor metamaterial response

- Achieve optical metamaterials - reduce losses in metamaterials

- Achieve three-dimensional isotropic left-handed metamaterials

- Create switchable and tunable metamaterials

- Devise/analyze new designs and approaches for negative index behaviour (chiral or anisotropic metamaterials)

- To explore novel phenomena and possibilities in metamaterials 


\section{To achieve optical left-handed materials?}

Scaling down of structures demonstrated initially in microwaves or $\mathbf{T H z}$

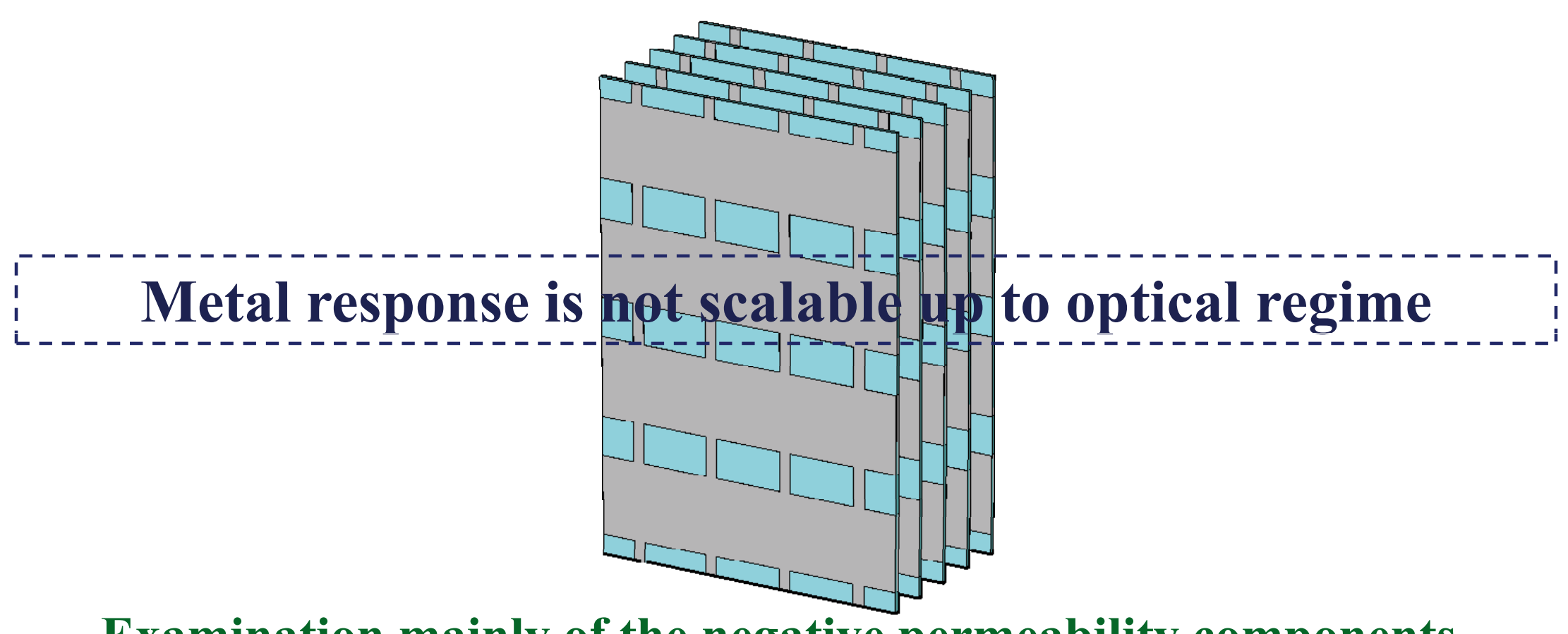

Examination mainly of the negative permeability components 


\section{Slab-pair: more suitable than SRR in sub-micron scale}
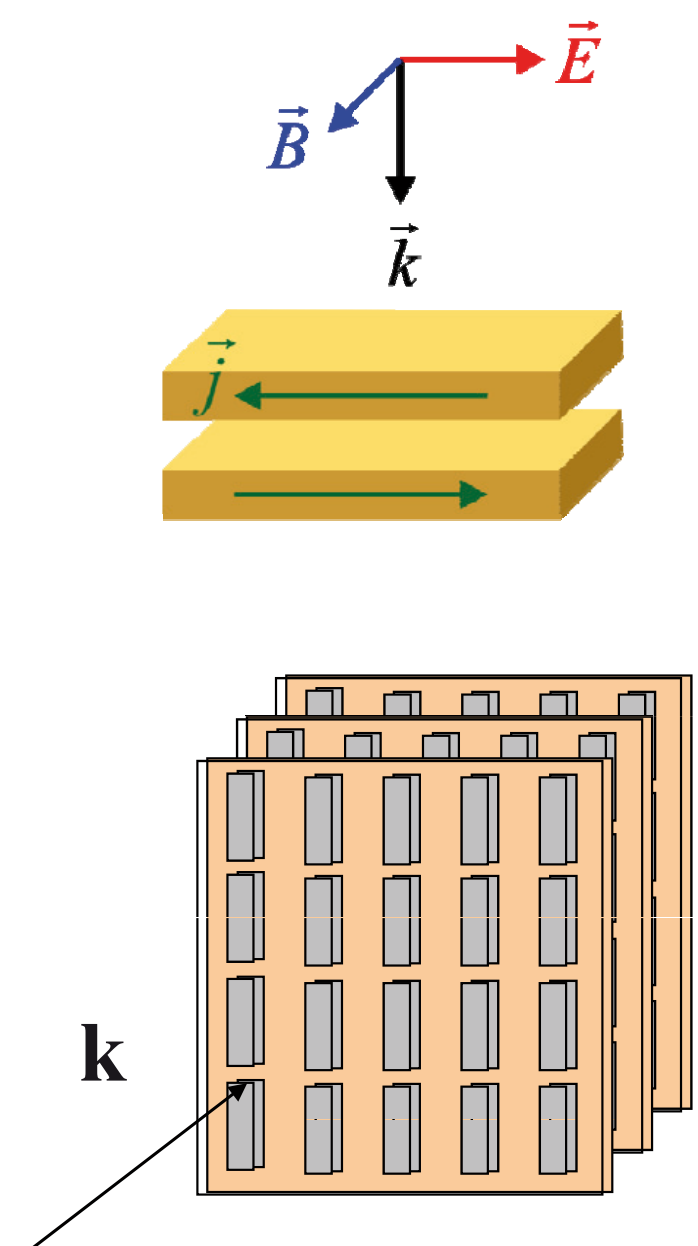

Shalaev et. al., Opt. Lett. 30, 3356 (2005)
- Magnetic response for normal incidence $\rightarrow$ exploitation in "small" length scales

-Simplified and easy in fabrication 


\section{Examples of slab-pair based left-handed materials}

Slabs \& wires

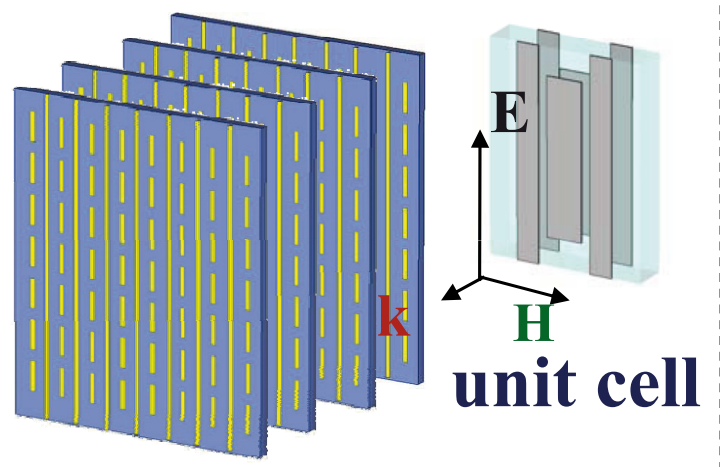

\section{Fishnet design}
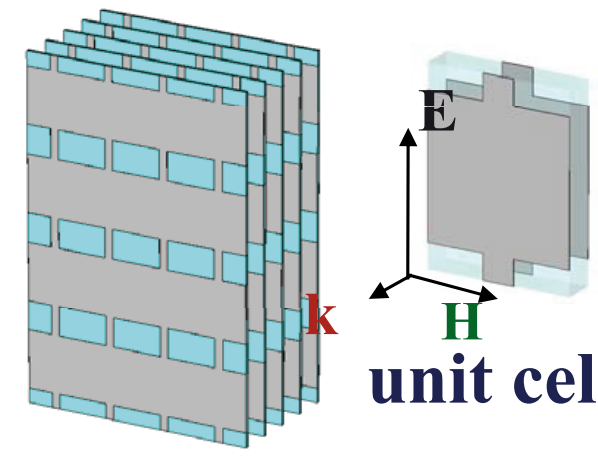
unit cell

Zhang, et. al., Opt. Expr. (2005) Ulrich (1966)
Slabs \& wires connected
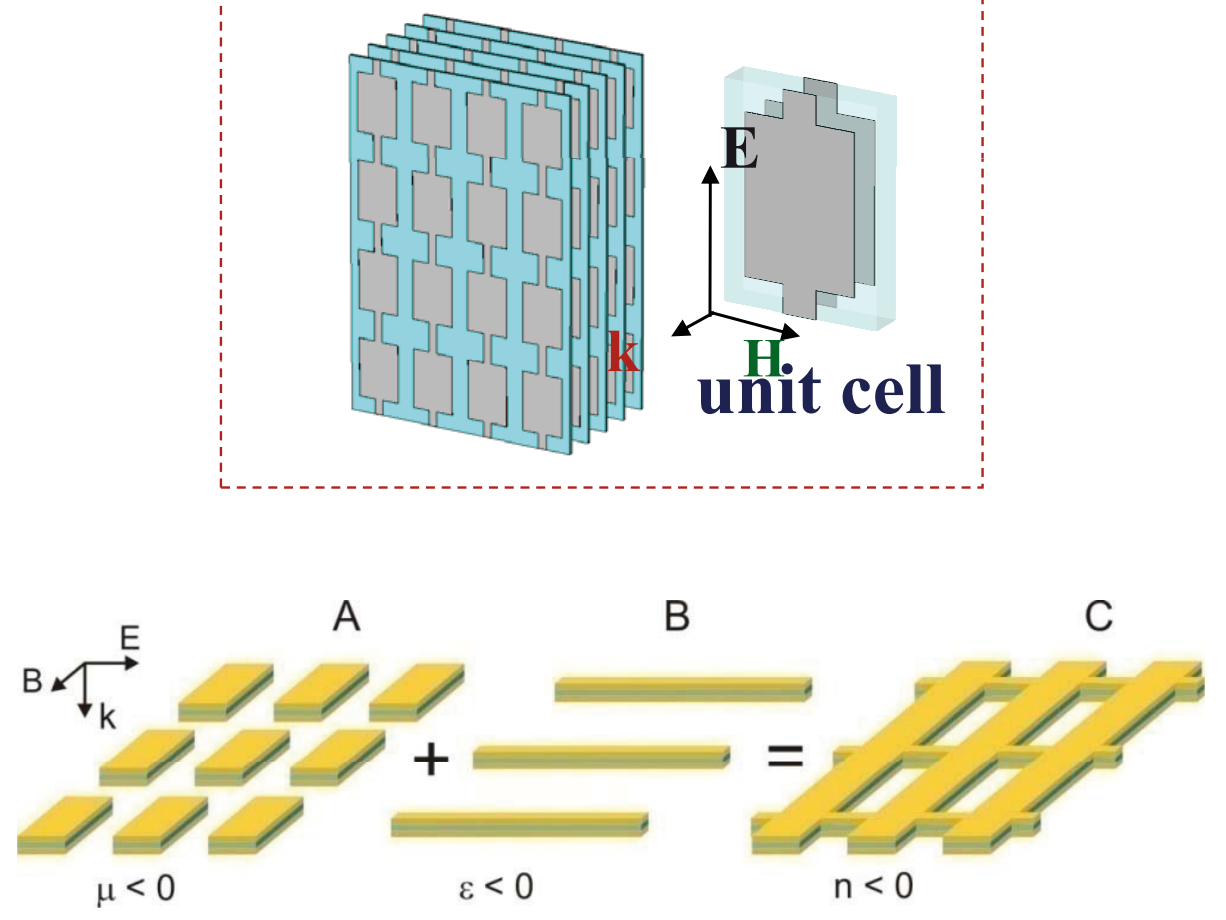

Fishnet (wide-slabs \& connected with wires): Optimized design for left-handed behavior 


\section{Negative $\boldsymbol{n}$ towards visible}

Fore review, see:

-Soukoulis et. al., Science 315 (2007)

-Shalaev, Nat. Mat. (2007)

Leading efforts by

-Karlsruhe

-Purdue

-Stuttgart

-Berkeley

$\bullet . .$. .

Lowest losses

$(\operatorname{Re}(n) / \operatorname{Im}(n)=3)$

$\checkmark$. det. al., Opt. Lett. (2007)

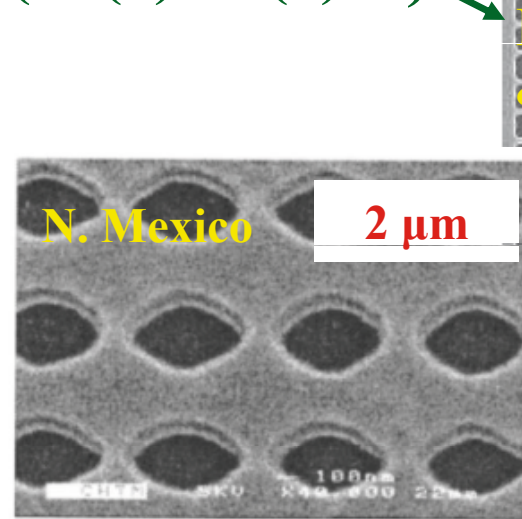
a olis: tarón: 8.

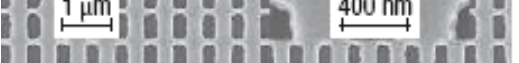
Zhang et. al., PRL (2005)
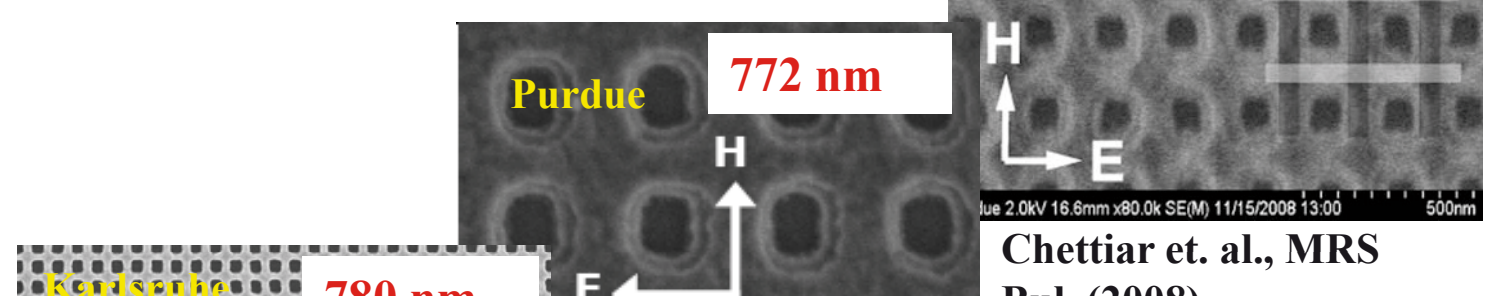

$18: 18 \div$ 1090400008000090008

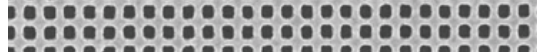

E - Bul. (2008)

\section{High losses}

Single functional layer 


\section{Optical metamaterials: Problems/challenges}

\section{High losses}

Limited fabrication capabilities

Current procedures:

-difficult/time-consuming

-expensive

- unable to produce

- complicated patterns

- large samples

- 3D isotropic designs 


\section{Optical metamaterials: Facing the challenges}

\section{High losses}

Limited fabrication capabilities

Current procedures:

-difficult/time-consuming

-expensive

- unable to produce

- complicated patterns

- large samples

- 3D isotropic designs
-Analysis \& design optimization

•"Good" constituent media

-Gain media?

- Novel approaches (anisotropic media, chiral media, EIT)
- Advancement of fabrication procedures

- New fabrication methods (direct laser writing, nanoimprint lithography)

- New designs/approaches, adapted to fabrication capabilities 


\section{Optical metamaterials: Facing the challenges}

\section{High losses}

Limited fabrication capabilities

Current procedures:

-difficult/time-consuming

-expensive

- unable to produce

- complicated patterns

- large samples

- 3D isotropic designs
-Analysis \& design optimization

- "Good" constituent media

-Gain media?

-Novel approaches (anisotropic media, chiral media, EIT)
- Advancement of fabrication procedures

- New fabrication methods (direct laser writing, nanoimprint lithography)

- New designs/approaches, adapted to fabrication capabilities 


\section{Optical metamaterials: Facing the challenges}

\section{High losses}

Limited fabrication capabilities

Current procedures:

-difficult/time-consuming

-expensive

- unable to produce

- complicated patterns

- large samples

- 3D isotropic designs
- Analysis \& design optimization

"Good" constituent media

-Gain media?

-Novel approaches (anisotropic media, chiral media, EIT)
- Advancement of fabrication procedures

- New fabrication methods (direct laser writing, nanoimprint lithography)

- New designs/approaches, adapted to fabrication capabilities 


\section{Designs discussed here}

Unit cells
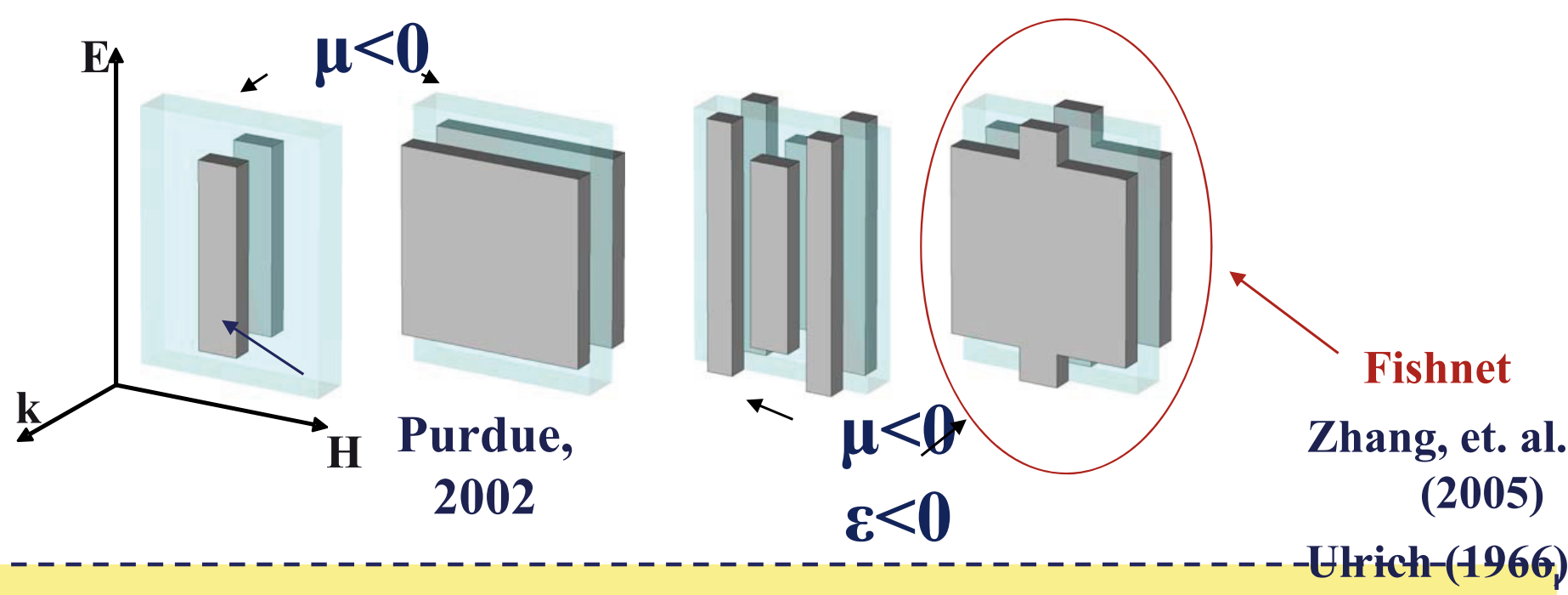

$\underline{\text { Aim }}$

Examine the behavior of the designs as they are scaled down targeting optical negative index response

Seek for optimization rules 


\section{Slab-pair magnetic response}

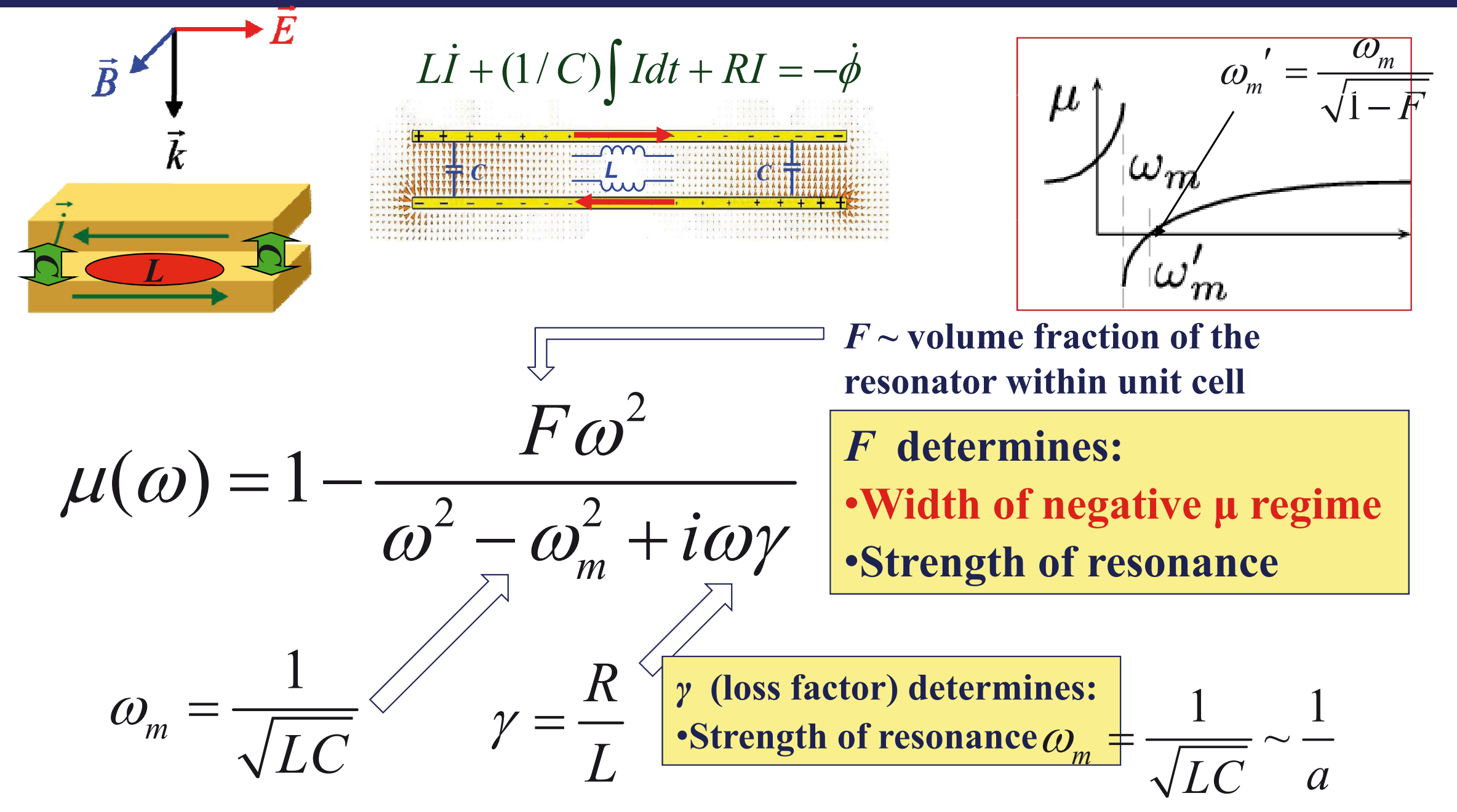

For uniform scaling:
a: lattice constant 


\section{Magnetic resonance frequency vs length scale}

Al metal, Glass substrate
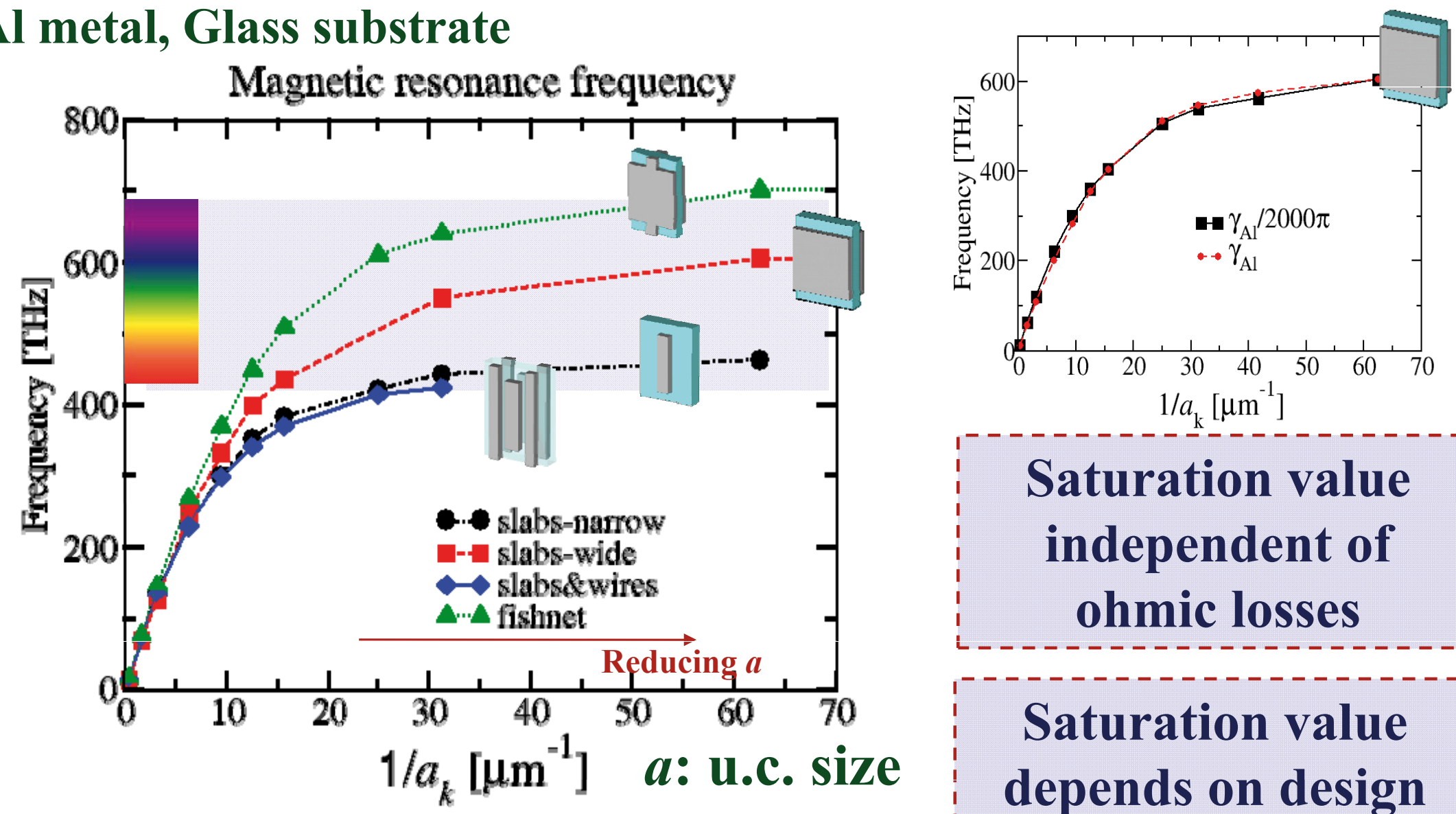

Saturation value independent of ohmic losses

Saturation value depends on design Penciu et. al., Phys. Rev. B 81, 235111 (2010)

Saturation of magnetic resonance frequency in small length scales $(a<500 \mathbf{n m})$ 


\title{
Magnetic permeability by scaling down the structures
}

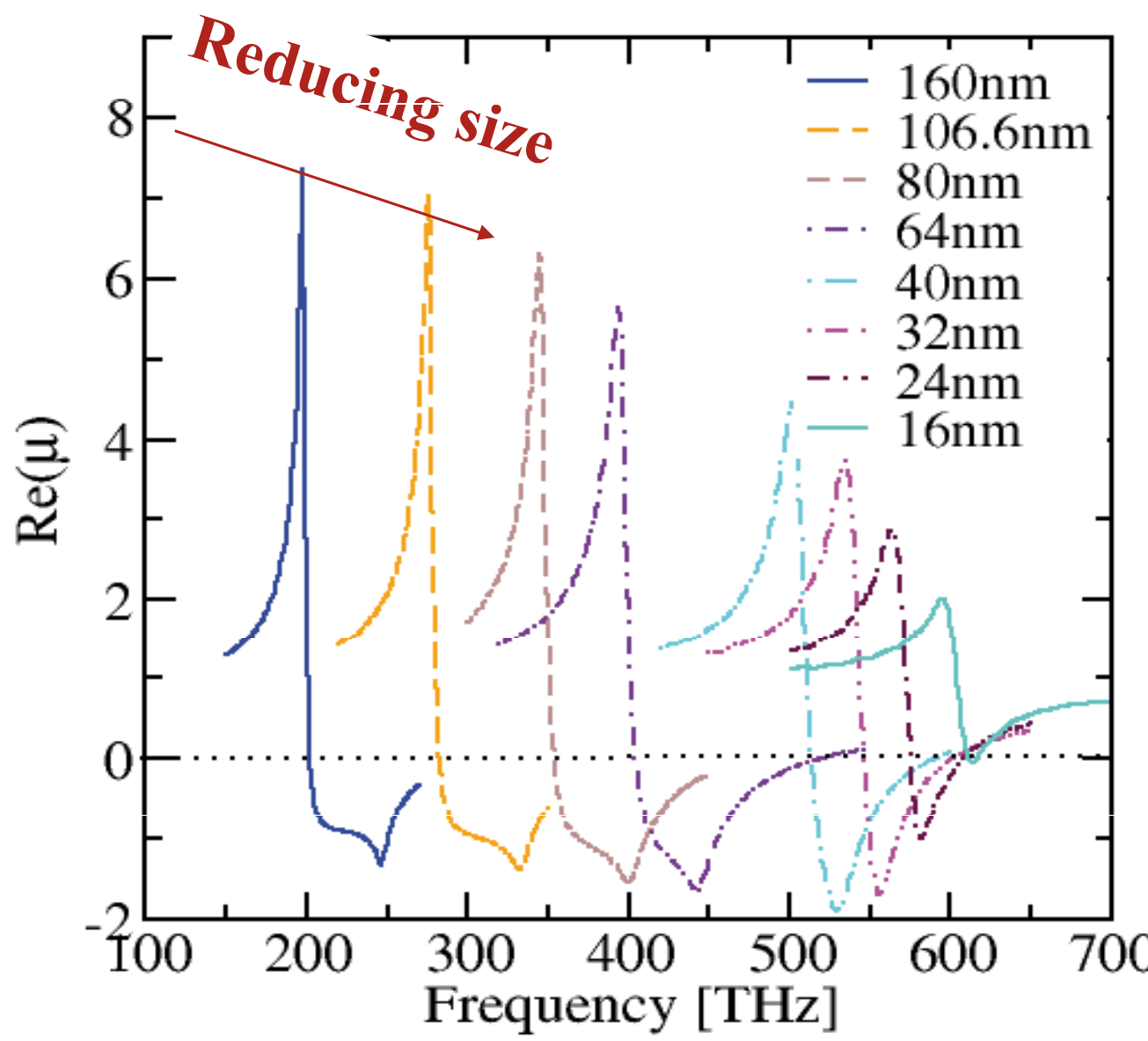

Al metal

Glass substrate

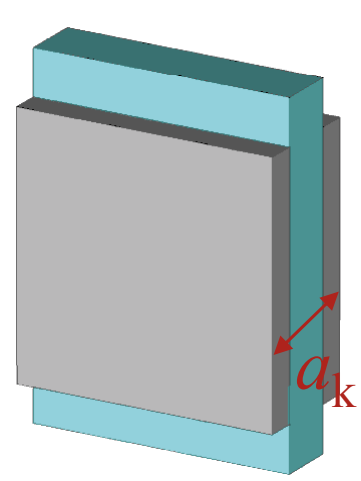

Penciu et. al., Phys. Rev. B 81, 235111 (2010)

\section{Weakening of magnetic resonance at small scales}

\author{
$\mu$ ultimately does not reach negative values
}




\section{Spectral width of negative $\boldsymbol{\mu}$ regime}

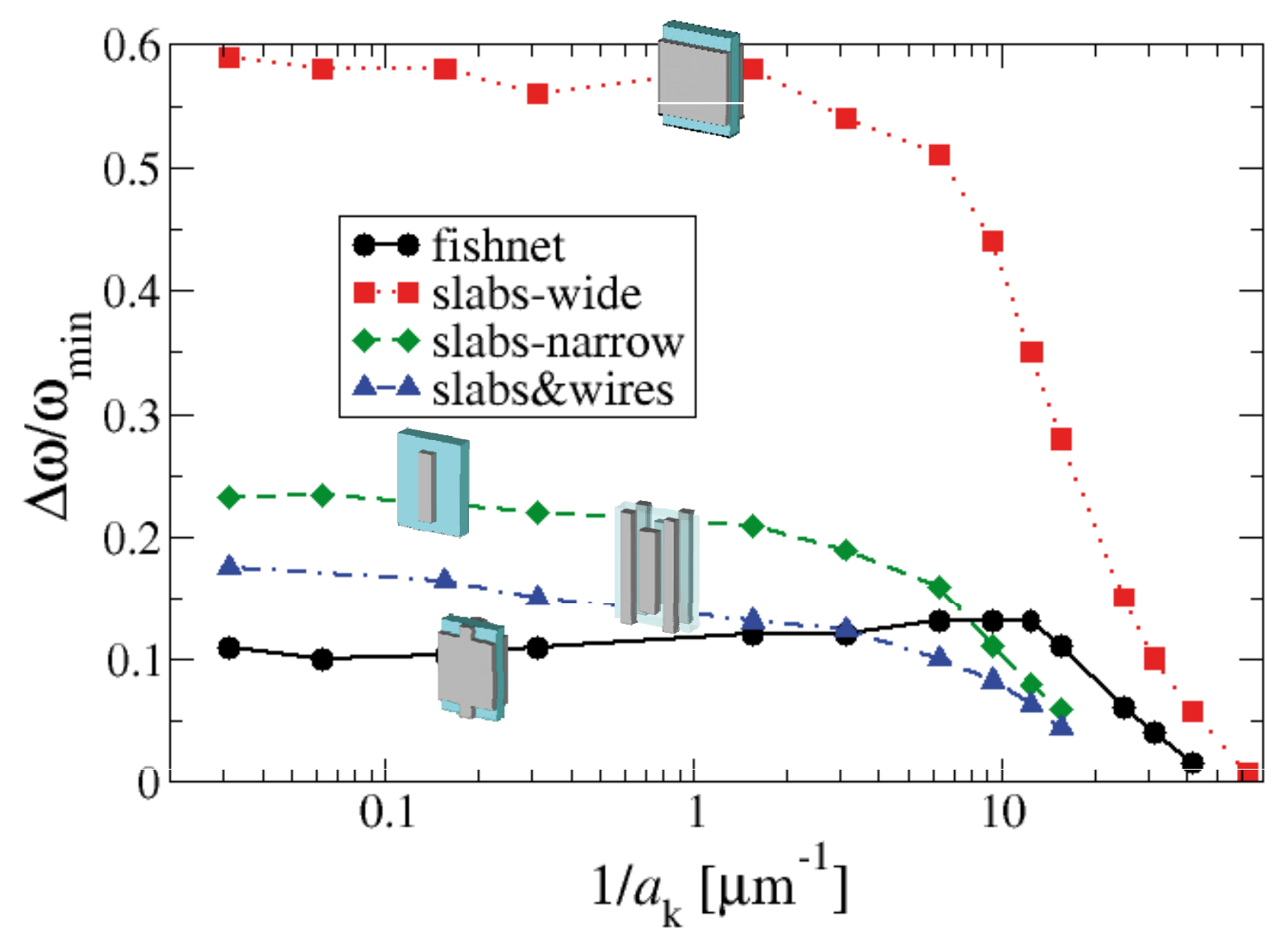

Al metal

Glass substrate

Penciu et. al., Phys. Rev. B 81, 235111 (2010)

Spectral width only slightly affected by metal loss

$\Delta \omega / \omega_{\min }:$ constant at larger scales

tends to zero for smaller scales 


\section{Losses by scaling down the structures}

Wide cut-slabs

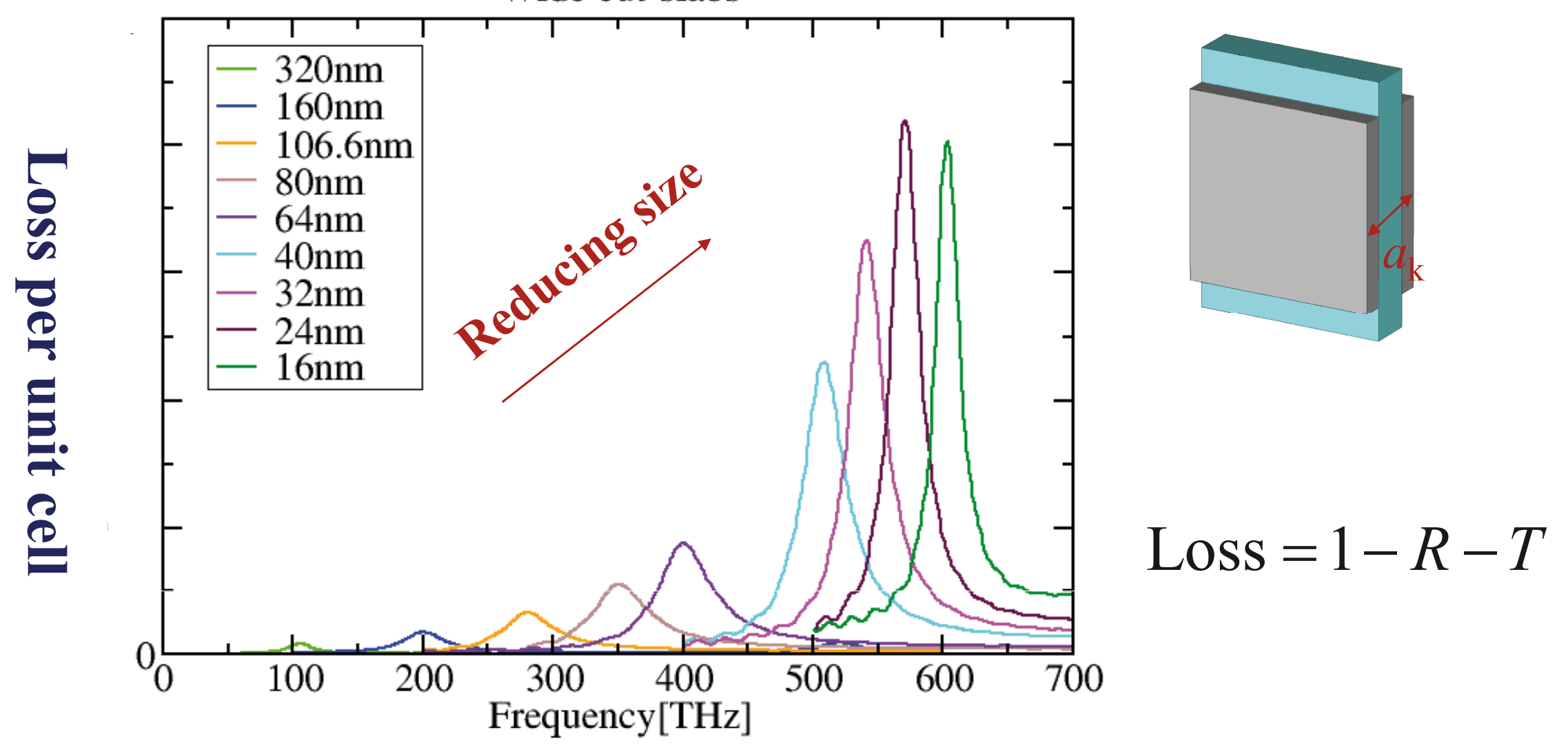

Increase of losses going to smaller scale

Penciu et. al., Phys. Rev. B 81, 235111 (2010) 


\section{Explaining $\omega_{\mathrm{m}}$ saturation and $\mu$-strength reduction}

Consideration of metal dispersive response in the conductivity:

$$
\sigma=i \varepsilon_{0} \frac{\omega_{p}^{2}}{\omega+i \gamma_{m}} \quad \begin{aligned}
& \text { Zhou et. al., 2005 } \\
& \text { Tretyakov, 2007 } \\
& \text { Solymar, 1976 } \\
& \text { Shvets et. al., 2005 }
\end{aligned}
$$

$$
R_{t o t}=\frac{1}{\sigma} \frac{l}{S}=\frac{\gamma_{m}}{\varepsilon_{0} \omega_{p}^{2}} \frac{l}{S}-i \omega \frac{1}{\varepsilon_{0} \omega_{p}^{2}} \frac{l}{S}=R-i \omega L_{\vartheta}
$$

$\omega_{\mathrm{p}}=$ metal plasma frequency

Inductive term (electrons inductance)

$\gamma_{\mathrm{m}}=$ metal collision frequency due to electrons inertia ("Difficulty" to accelerate finite mass particles with such high rates)

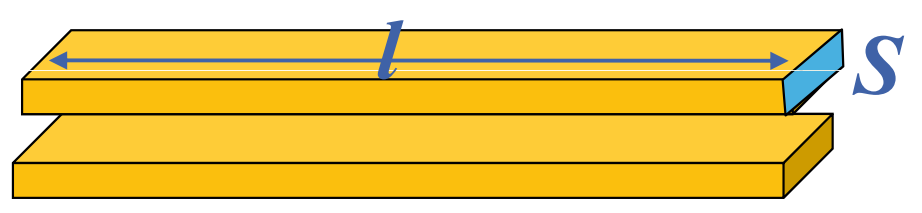

$E_{\text {kinetic }}=\frac{1}{2} L_{e} I^{2}$

$$
m \frac{d \mathbf{v}}{d t}+\gamma_{m} \mathbf{v}=q \mathbf{E}
$$




\section{Slab-pair effective permeability in sub- $\mu \mathrm{m}$ scale}

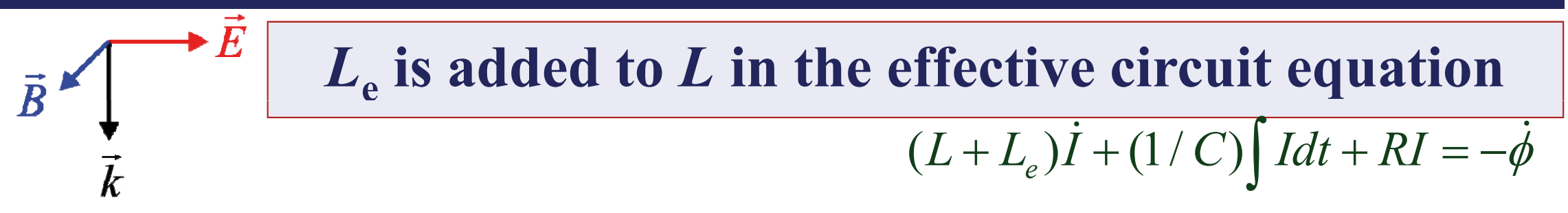

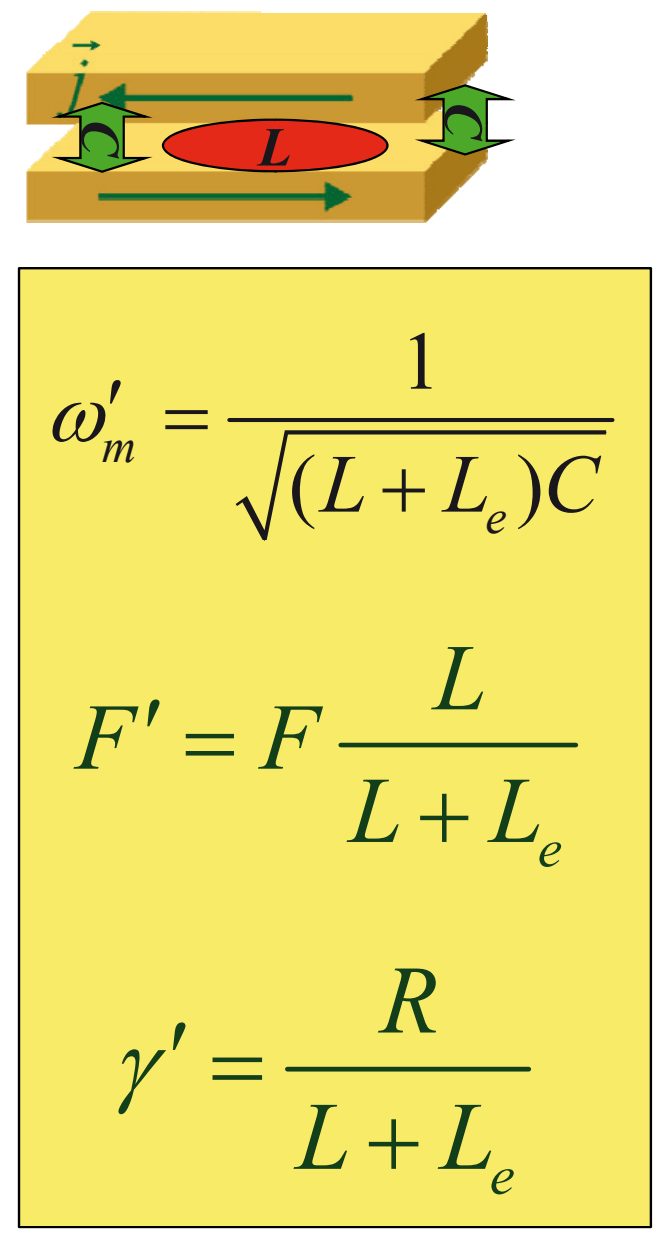

$$
\mu(\omega)=1-\frac{F^{\prime} \omega^{2}}{\omega^{2}-\omega_{m}^{\prime 2}+i \omega \gamma^{\prime}}
$$

$F \sim$ volume fraction of the resonator within unit cell

$$
\begin{gathered}
L_{e}=\frac{1}{\varepsilon_{0} \omega_{p}^{2}} \frac{l}{S} \\
R=\frac{\gamma_{m}}{\varepsilon_{0} \omega_{p}^{2}} \frac{l}{S}
\end{gathered}
$$

For uniform scaling:

$$
\begin{array}{rlrl}
L_{e} & \sim \frac{1}{a} & R \sim \frac{1}{a} \\
L & \sim a & C \sim a
\end{array}
$$

a: lattice constant 


\section{Explaining the observed response}

\section{Magnetic resonance} frequency saturates to $\omega_{\mathrm{m} \text {-max }}$ -dependent on shape -independent of ohmic losses -proportional to metal plasma frequency

$$
L \propto a, C \propto a, L_{e} \propto 1 / a, R \propto 1 / a
$$

a: u.c. size

$$
\omega_{m}^{\prime}=\frac{1}{\sqrt{\left(L+L_{e}\right) C}} \sim \frac{1}{\sqrt{a^{2}+c_{1}}} \rightarrow \text { const. }
$$

Strength parameter $\boldsymbol{F}^{\prime}$ becomes $\begin{aligned} & \text { Strength parameter } \boldsymbol{F}^{\prime} \text { becomes } \\ & \text { proportional to area } \rightarrow \text { Vanishing of } \\ & \text { negative } \boldsymbol{\mu} \text { regime even if the absence }\end{aligned} \quad F^{\prime}=F \frac{L}{L+L_{e}} \rightarrow F \frac{a^{2}}{a^{2}+1}$ of ohmic losses

Loss parameter increases for small length scales; $-\gamma^{\prime}$ depends on shape $\gamma^{\prime}=\frac{R}{L+L_{e}}=\frac{\gamma_{m}}{1+L / L_{e}} \rightarrow \frac{\gamma_{m}}{1+a^{2}}$ -it saturates to metal collision frequency Penciu et. al, Phys. Rev. B 81,235111 (2010) 


\section{For high frequency magnetic metamaterials}

$\underbrace{\text { High }}_{m}=\frac{1}{\sqrt{\left(L+L_{e}\right) C}}$

for

High operation

frequency

Broad-band

Low-loss

High $F^{\prime}=F \frac{1}{1+L_{e} / L}$

$\mu=\mu_{0}\left[1-\frac{F^{\prime} \omega^{2}}{\omega^{2}-\omega_{m}^{2}+i \omega \gamma^{\prime}}\right]$

\section{Requirements}
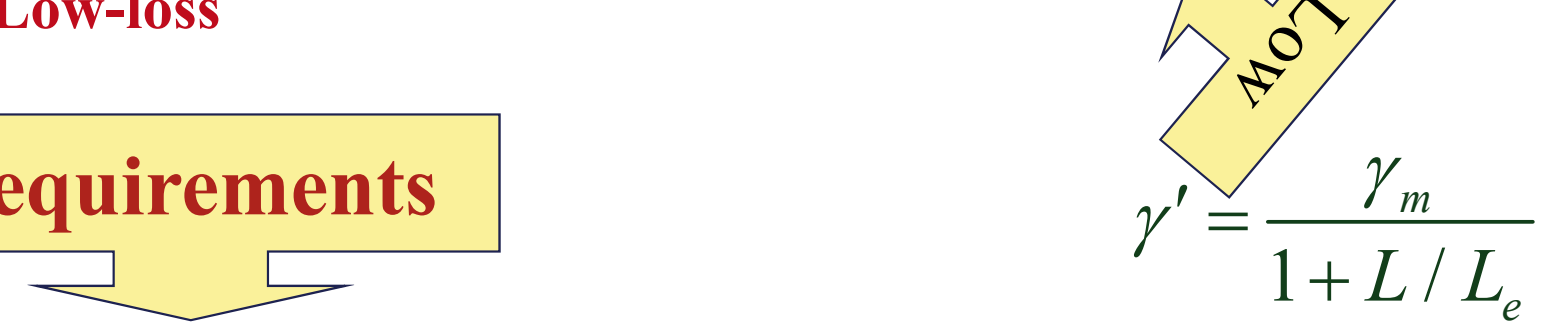

- Small capacitance, $C$

-Small $L_{\mathrm{e}}$

- Large metal plasma

frequency - small collision frequency

- $L$ ?: opposite role in $\omega_{\mathrm{m}}$ and $\mu$ 


\section{Electrons inductance at the electric dipole resonance: Resonance frequency}

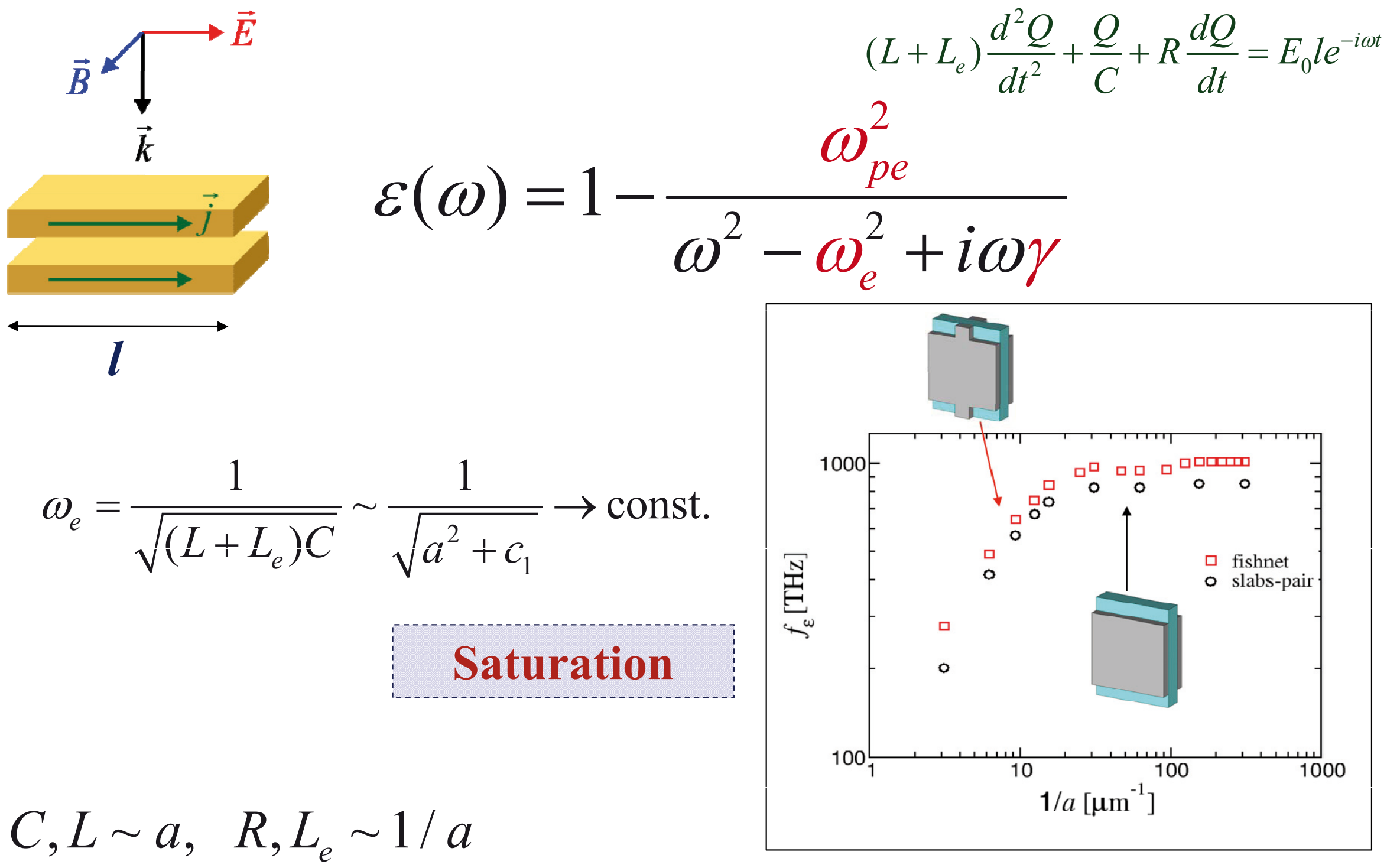




\section{Electrons inductance at the electric dipole resonance: Resonance strength}

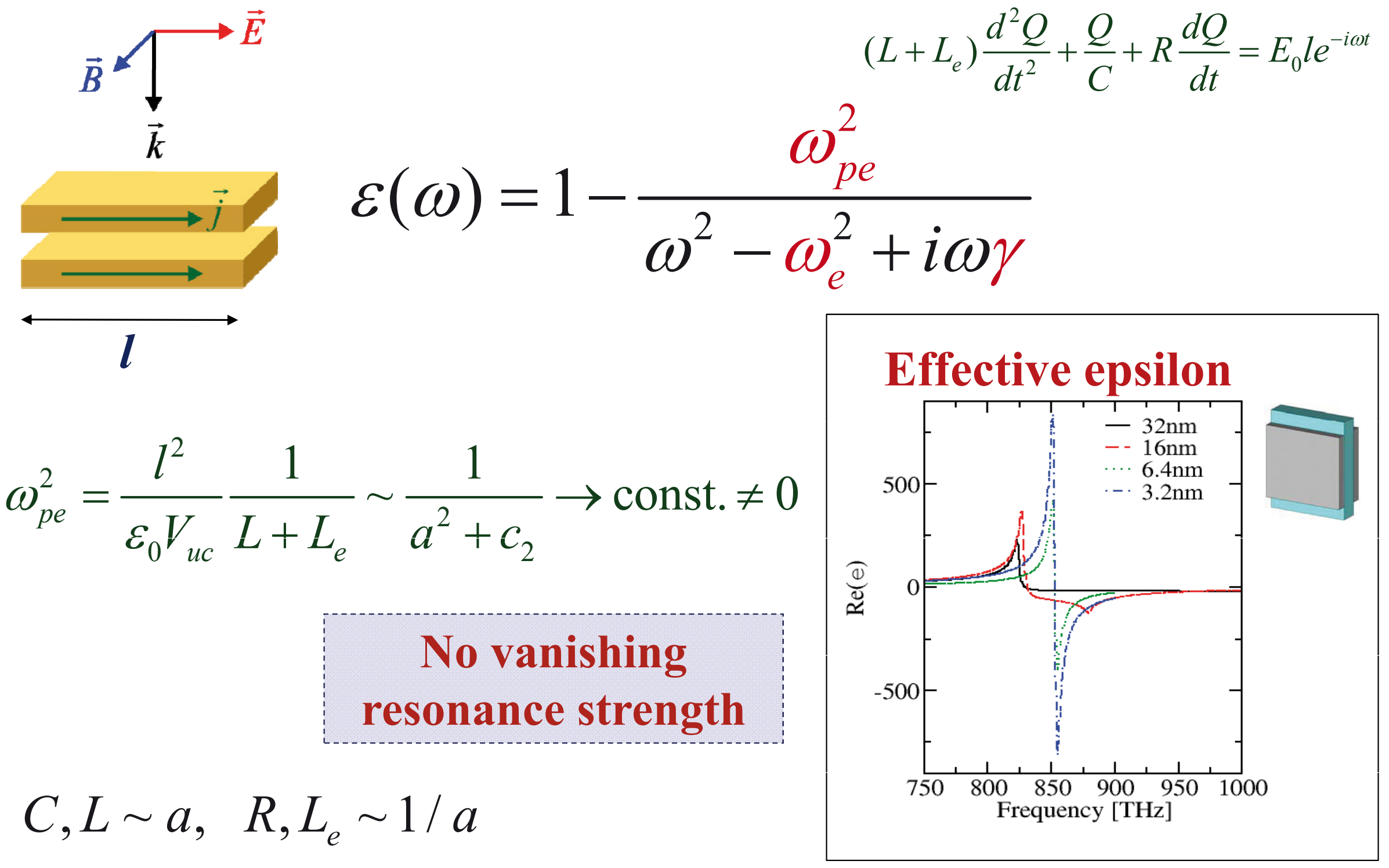




\section{Electrons inductance at the electric dipole resonance features?}

$$
\begin{aligned}
& \vec{B} \underset{\vec{k}}{\longrightarrow} \vec{E} \\
& \stackrel{\longrightarrow}{\longrightarrow} \\
& \varepsilon(\omega)=1-\frac{\omega_{p e}^{2}}{\omega^{2}-\omega_{e}^{2}+i \omega \gamma} \\
& \omega_{e}=\frac{1}{\sqrt{\left(L+L_{e}\right) C}} \sim \frac{1}{\sqrt{a^{2}+c_{1}}} \rightarrow \text { const. } \\
& \omega_{p e}^{2}=\frac{l^{2}}{\varepsilon_{0} V_{u c}} \frac{1}{L+L_{e}} \sim \frac{1}{a^{2}+c_{2}} \rightarrow \text { const. } \neq 0
\end{aligned}
$$

\section{Saturation}




\section{Optical metamaterials: Facing the challenges}

\section{High losses}

Limited fabrication capabilities

Current procedures:

-difficult/time-consuming

-expensive

- unable to produce

- complicated patterns

- large samples

- 3D isotropic designs
-Analysis \& design optimization

- "Good" constituent media

-Gain media?

-Novel approaches (anisotropic media, chiral media, EIT)
- Advancement of fabrication procedures

- New fabrication methods (direct laser writing, nanoimprint lithography)

- New designs/approaches, adapted to fabrication capabilities 


\section{"Connected" structures for direct laser writing}

$1 D$

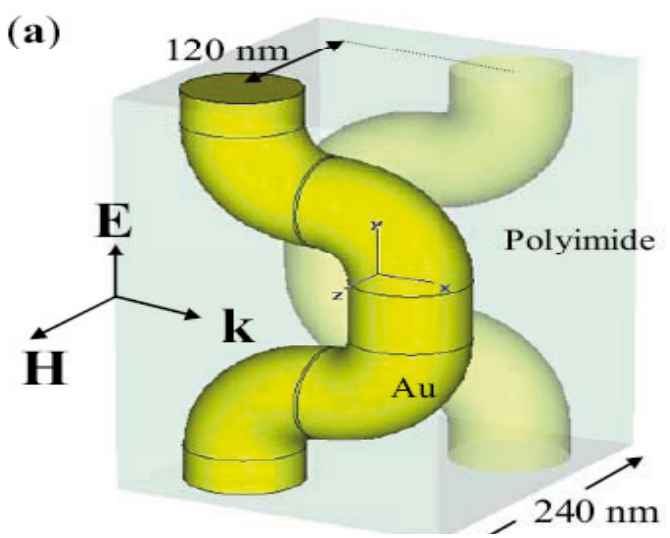

(b)

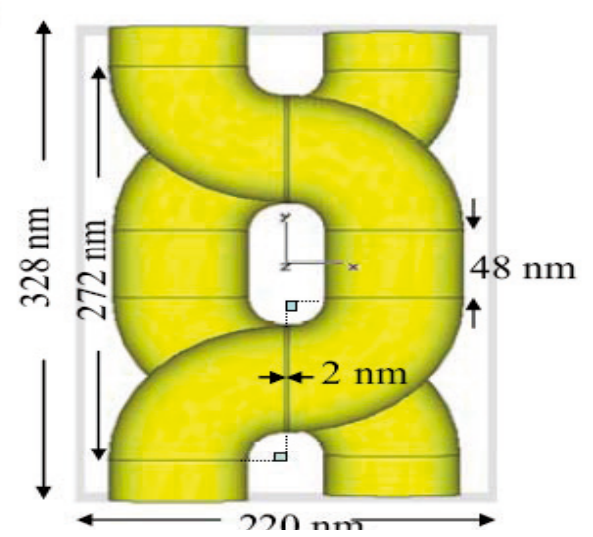

Gold in

polyimide

$\operatorname{Re}(n) /\left.\operatorname{Im}(n)\right|_{n=-1}=5$

Double negative@ $\sim 200$ THz (1.5 $\mu \mathrm{m})$
2D

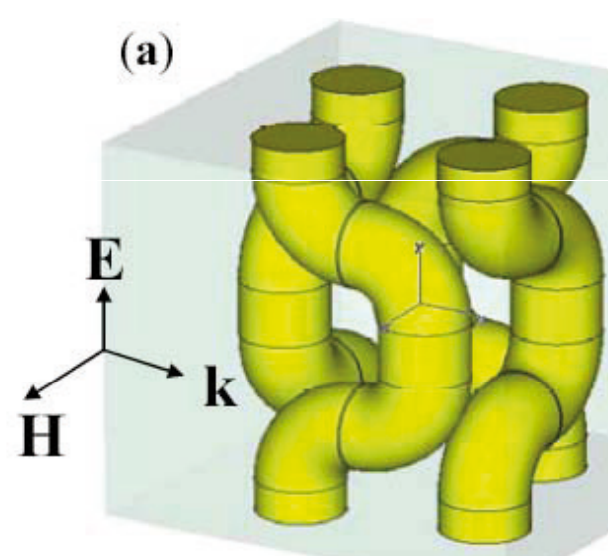

Calculations by D. Guney (b)

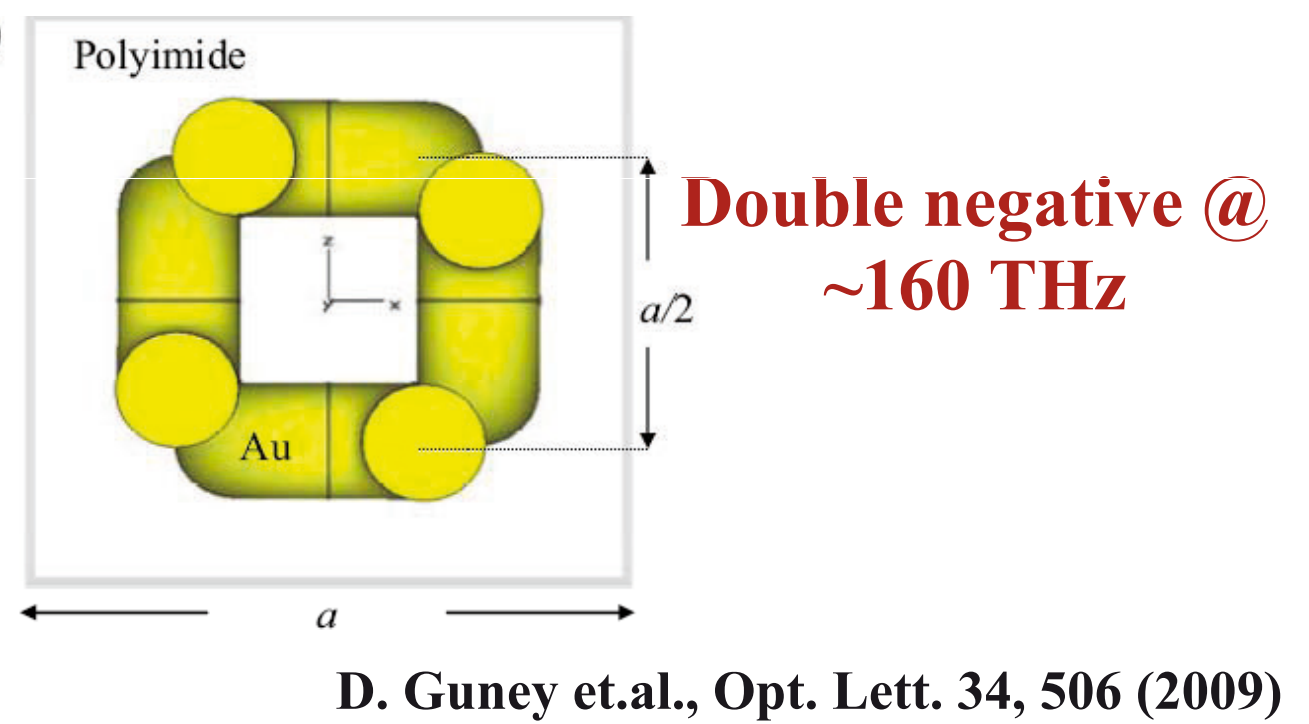




\section{"Connected" structure realization}

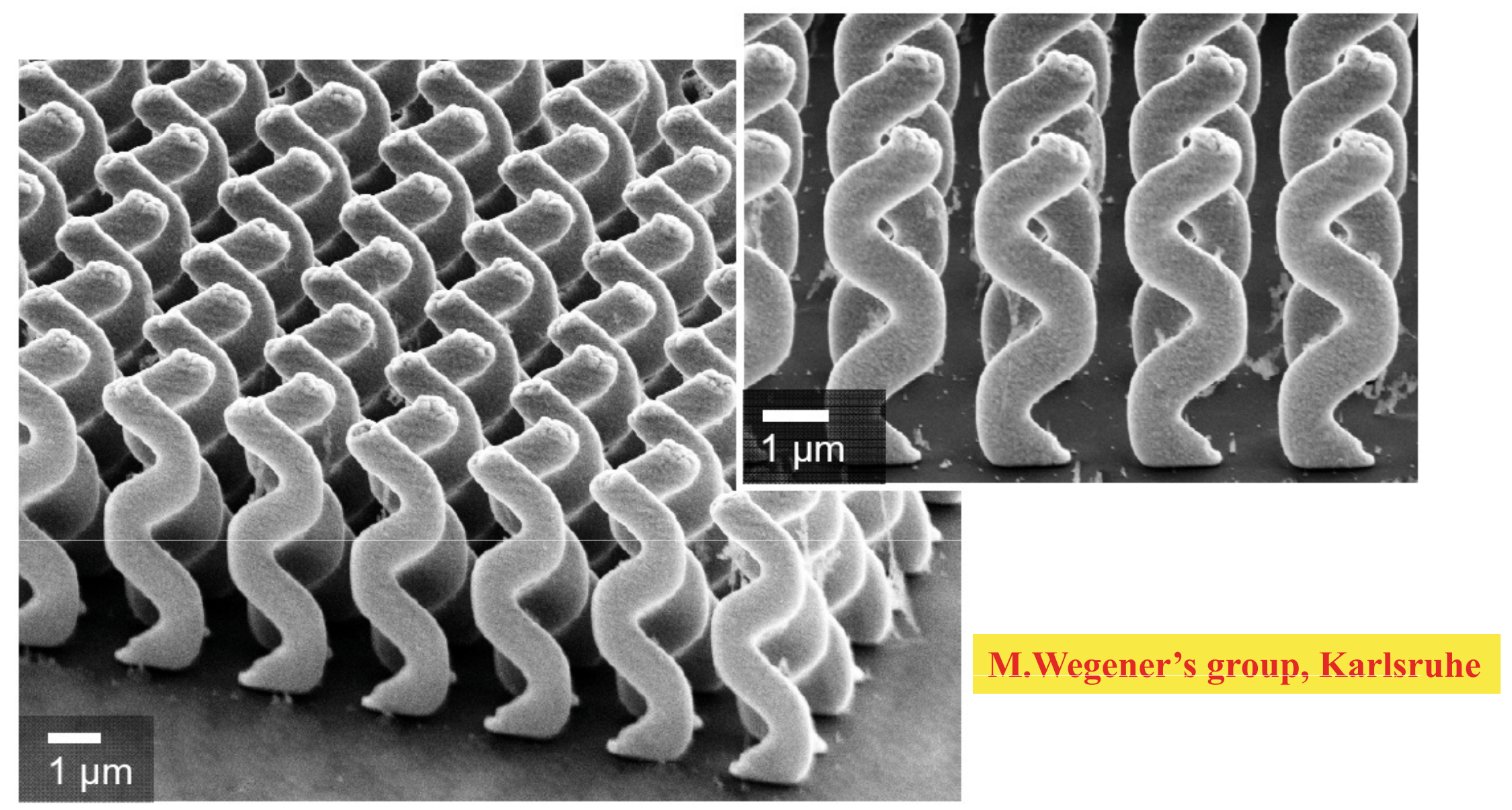




\section{Optical metamaterials: Facing the challenges}

\section{High losses}

Limited fabrication capabilities

Current procedures:

-difficult/time-consuming

-expensive

- unable to produce

- complicated patterns

- large samples

- 3D isotropic designs
-Analysis \& design optimization

- "Good" constituent media

-Gain media?

-Novel approaches (anisotropic media, chiral media, EIT)

- Advancement of fabrication procedures

- New fabrication methods (direct laser writing, nanoimprint lithography)

- New designs/approaches, adapted to fabrication capabilities 


\section{Negative refractive index in chiral media}

Chiral structure: not-identical to its mirror image

-Different index for left- and right$n_{ \pm}=\sqrt{\varepsilon \mu} \pm \kappa \quad \begin{aligned} & \text { handed circularly polarized waves } \\ & \begin{array}{l}\text { Alternative path to achieve negative } \\ \text { index (Pendry, Tretyakov) }\end{array}\end{aligned}$

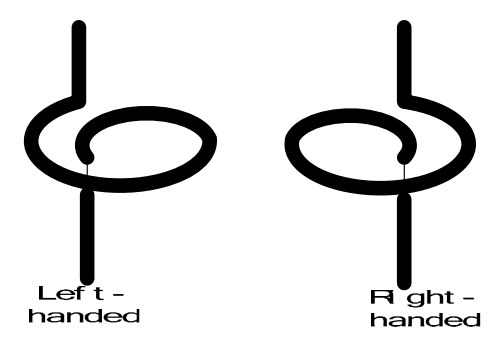

$$
\begin{aligned}
& \boldsymbol{D}=\varepsilon \boldsymbol{E}+i \kappa \boldsymbol{H} \\
& \boldsymbol{B}=\mu \boldsymbol{H}-i \kappa \boldsymbol{E}
\end{aligned}
$$

Besides negative index:

-Polarization rotation

-Circular dichroism
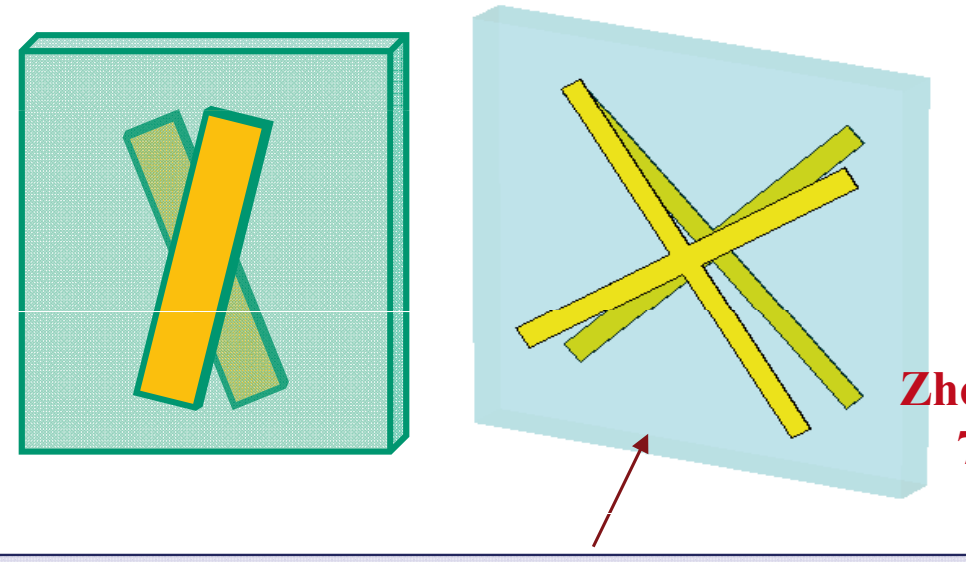

\section{Twisted}

cross

Zhou et. al., Phys. Rev. B 79, 121104R (2009)

Negative index

Large polarization rotation

Large circular dichroism 


\section{Chiral optical structures}

\section{Twisted gold crosses}

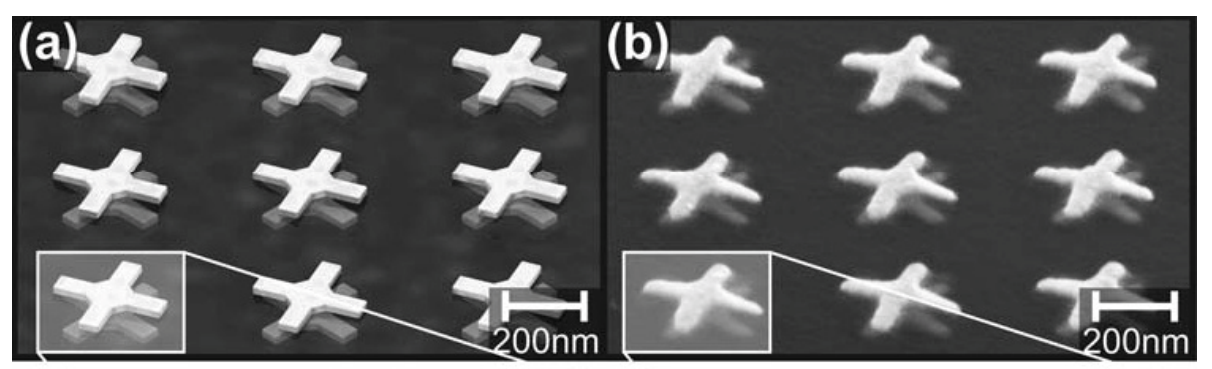

Response@1-2 $\mu \mathrm{m}$

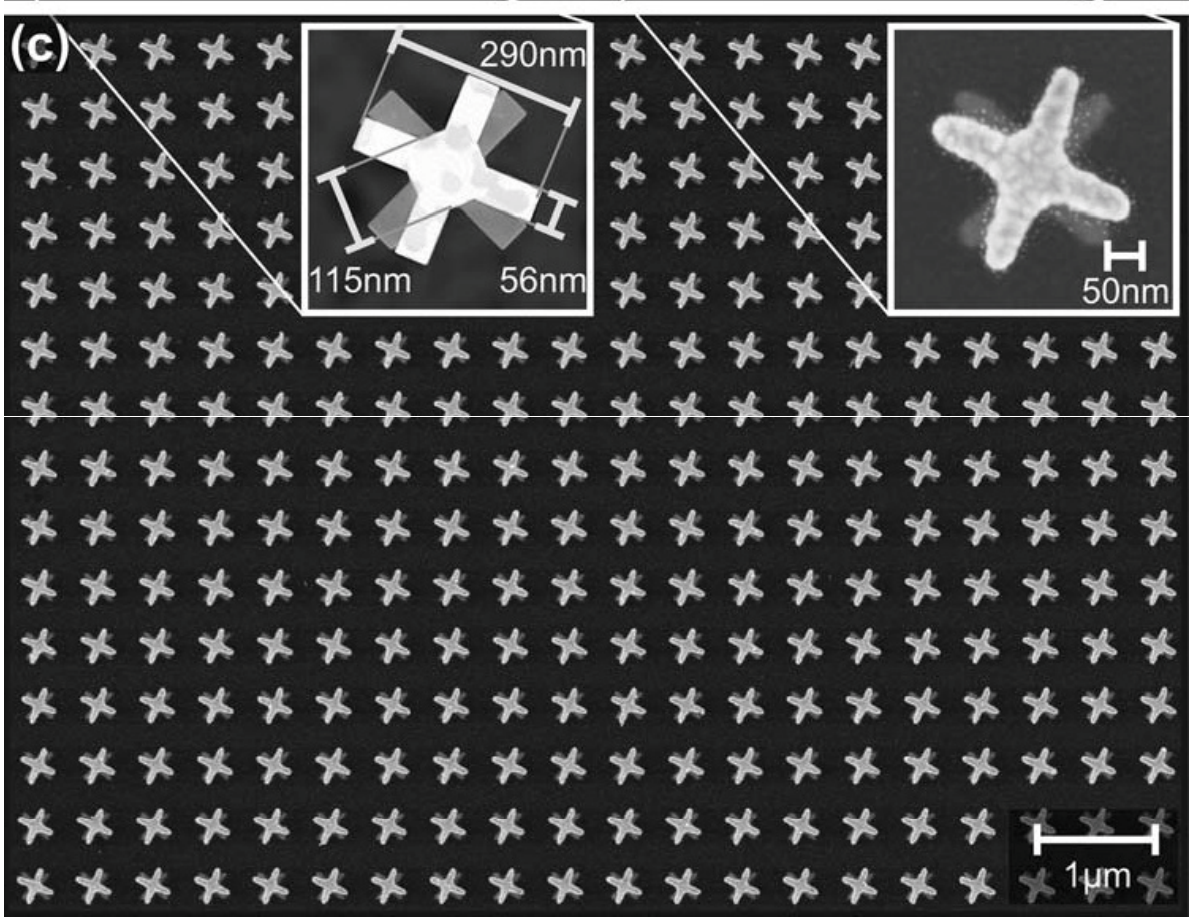

Large polarization rotation Large circular dichroism

M. Wegener's group, Opt. Lett. (2009) 


\section{Optical metamaterials: Facing the challenges}

\section{High losses}

Limited fabrication capabilities

Current procedures:

-difficult/time-consuming

-expensive

- unable to produce

- complicated patterns

- large samples

- 3D isotropic designs
-Analysis \& design optimization

- "Good" constituent media

-Gain media?

-Novel approaches (anisotropic media, chiral media, EIT)
- Advancement of fabrication procedures

- New fabrication methods (direct laser writing, nanoimprint lithography)

- New designs/approaches, adapted to fabrication capabilities 


\section{Superlenses for anisotropic media?}

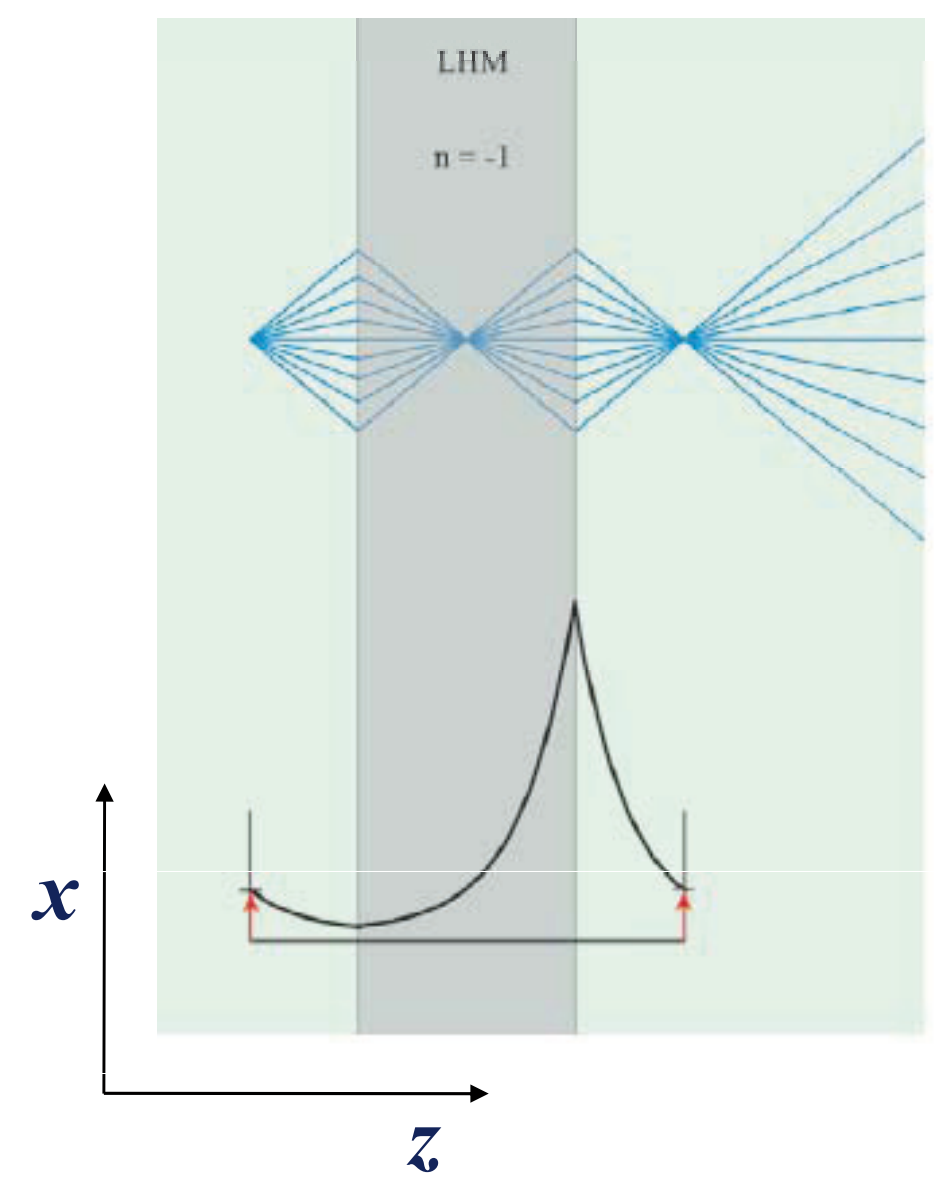

Perfect lensing conditions:

Propagating components:

Omnidirectional total transmission

Evanescent components:

Excitation of dispersionless surface plasmon modes

\section{Aim: Examine these conditions} for anisotropic materials

$$
\overline{\overline{\varepsilon_{2}}}=\left[\begin{array}{ccc}
\varepsilon_{2 x} & 0 & 0 \\
0 & \varepsilon_{2 y} & 0 \\
0 & 0 & \varepsilon_{2 z}
\end{array}\right], \overline{\overline{\mu_{2}}}=\left[\begin{array}{ccc}
\mu_{2 x} & 0 & 0 \\
0 & \mu_{2 y} & 0 \\
0 & 0 & \mu_{2 z}
\end{array}\right]
$$

Work done by N.H. Shen 


\section{Superlensing conditions for anisotropic media}

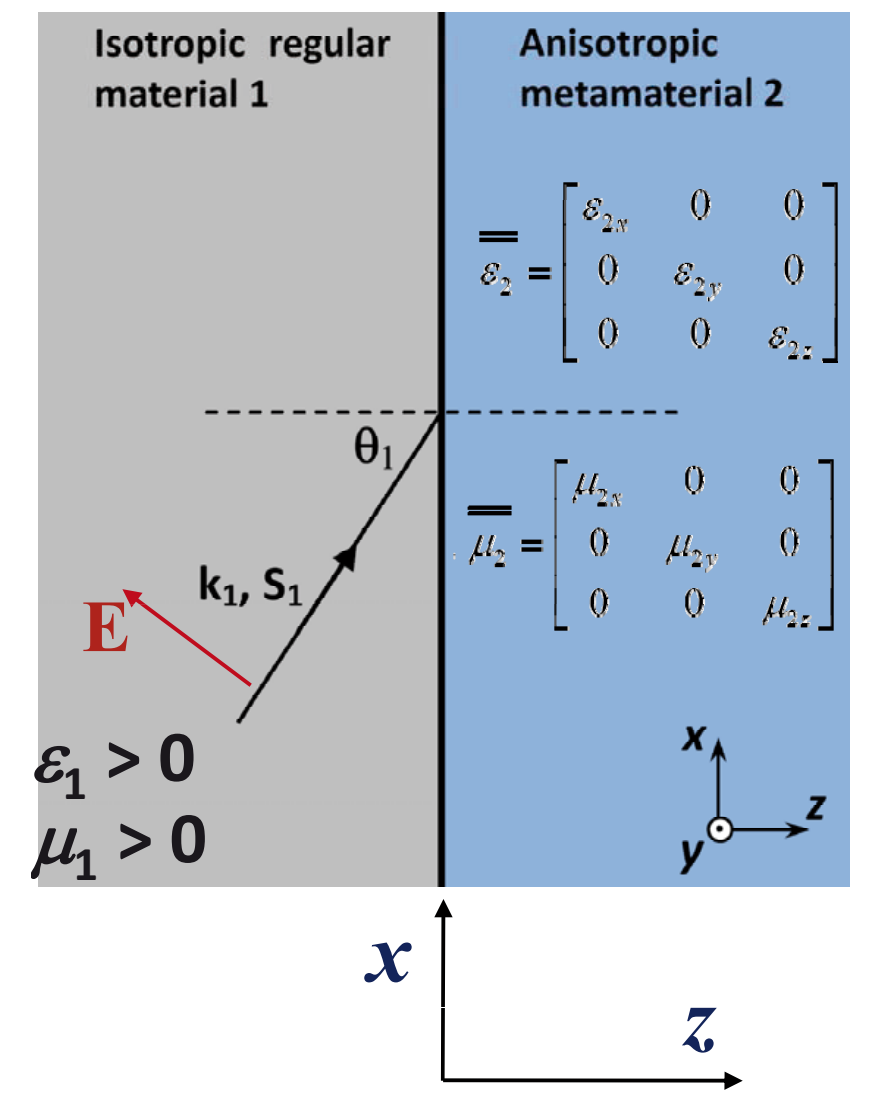

For p-polarization

$$
\begin{gathered}
\frac{\varepsilon_{2 x}}{\mu_{2 y}}=\frac{\varepsilon_{1}}{\mu_{1}} \quad \text { For isotropic media: } \\
\varepsilon_{2 x} \varepsilon_{2 z}=\varepsilon_{1}^{2} \quad \varepsilon_{2}=-\varepsilon_{1}, \mu_{2}=-\mu_{1} \\
\varepsilon_{2 x}, \varepsilon_{2 z}, \mu_{2 y}<0 \\
\begin{array}{c}
\text { Easy to implement } \\
\text { conditions with planar } \\
\text { technologies }
\end{array}
\end{gathered}
$$




\section{Lens formula for anisotropic lenses}

$$
d_{\text {Source }}+d_{\text {Image }}=\left(\varepsilon_{2 x} / \varepsilon_{1}\right) d
$$

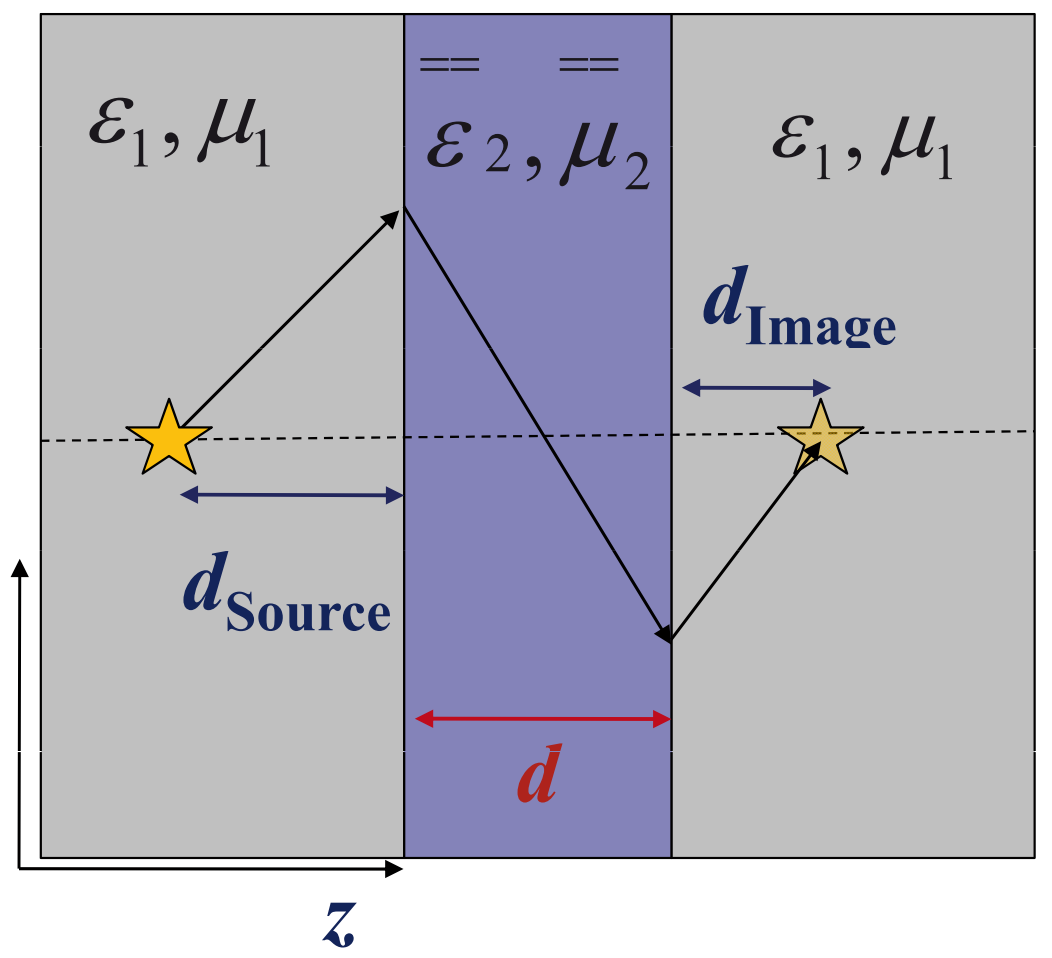

Possibility for thin lenses! (less influenced by losses) 


\section{Anisotropic "perfect" lens: Negative refraction \& focusing}

Negative refraction by an anisotropic double negative slab

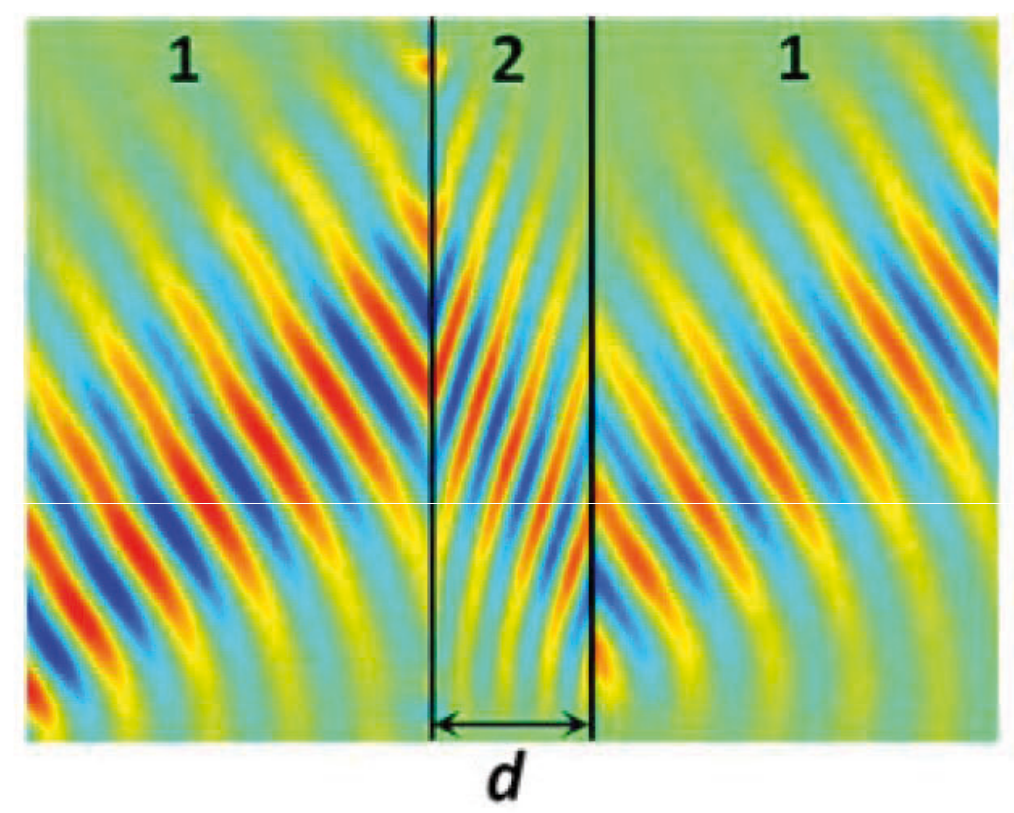

$\varepsilon_{2 x}=\mu_{2 y}=-2+0.01 i, \quad \varepsilon_{2 z}=-0.5+0.01 i$
Focusing in an anisotropic double negative slab

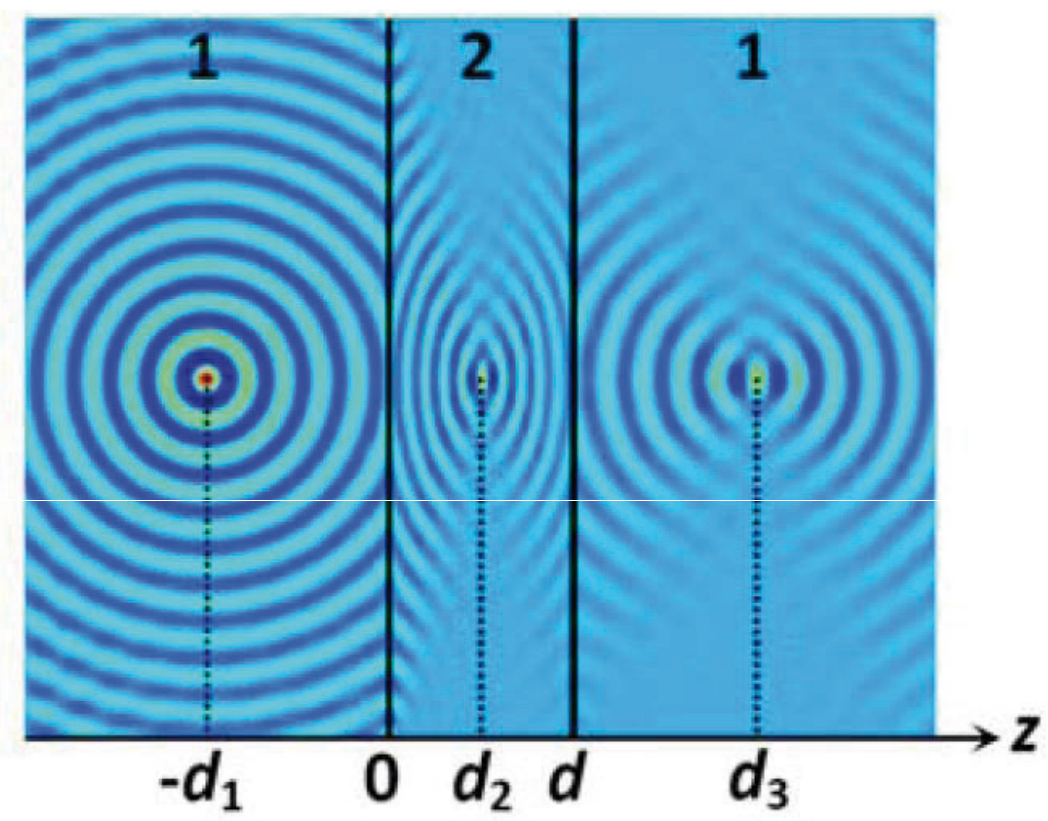

$d=0.2 \lambda \quad$ resolution $=\lambda / 5$ 


\section{Optical metamaterials: Facing the challenges}

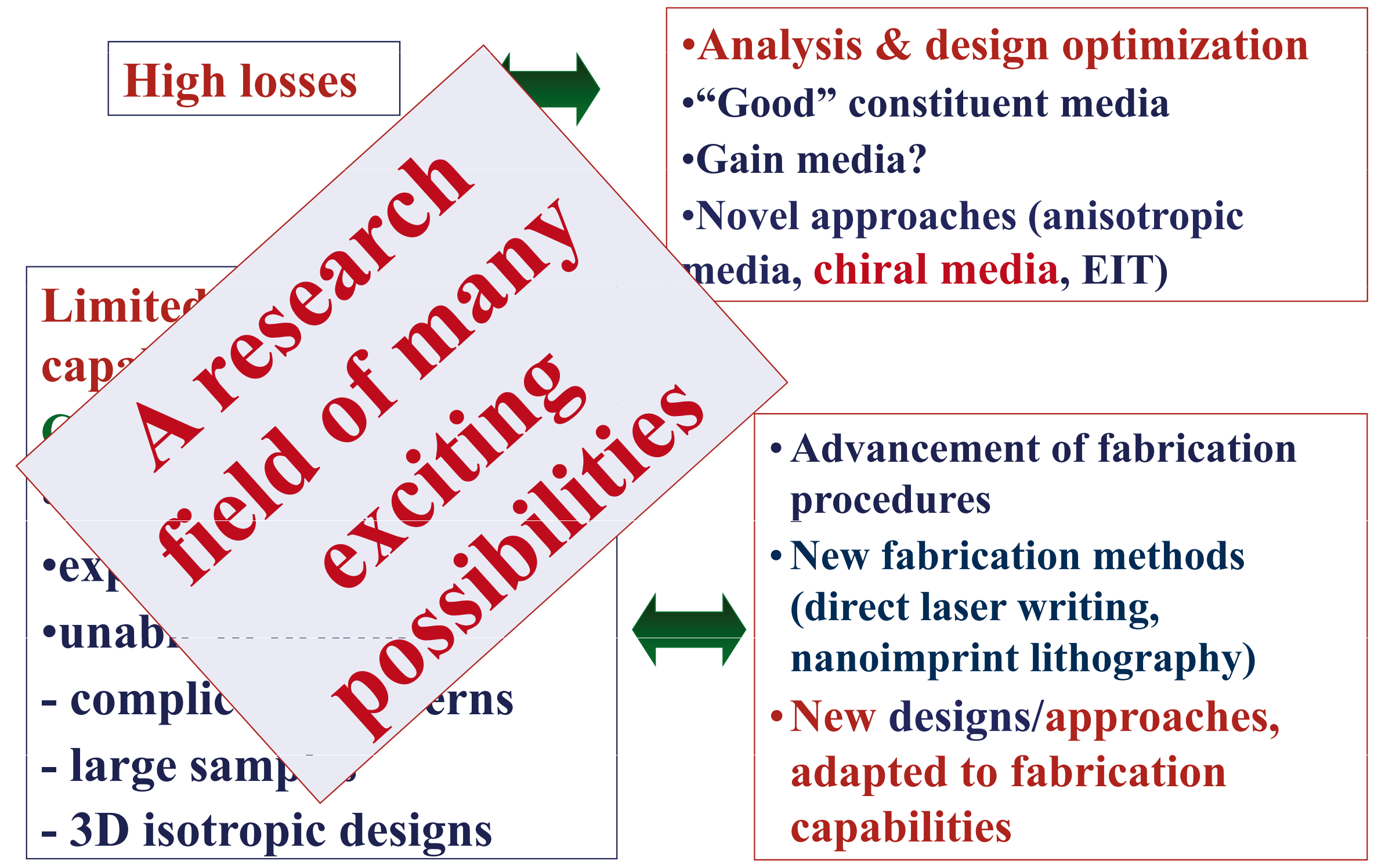




\section{Work thanks to}
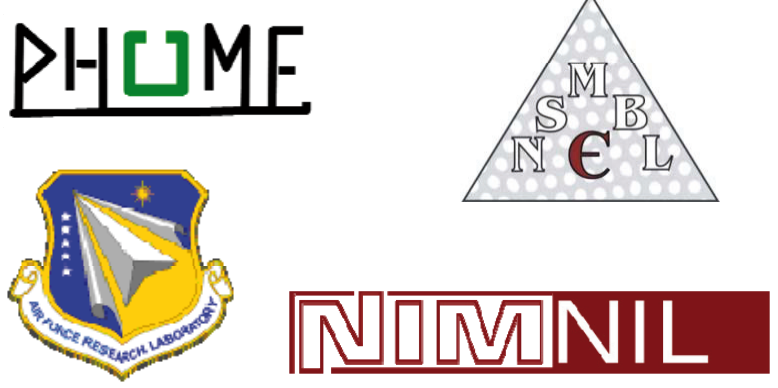

ECONAM

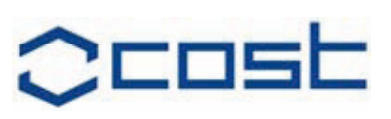

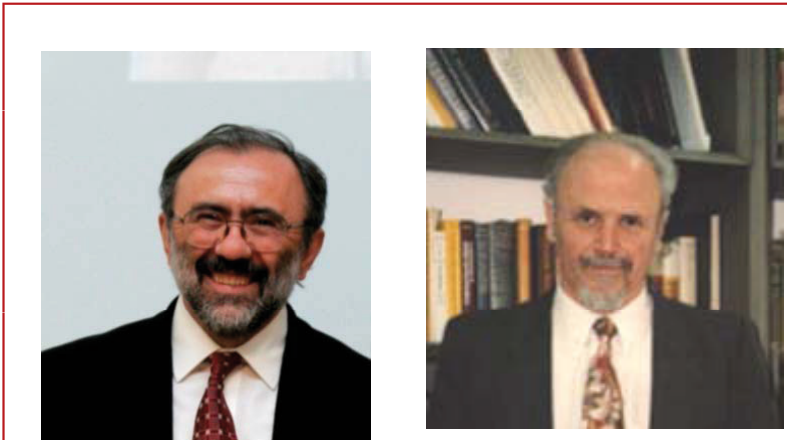

E. Economou

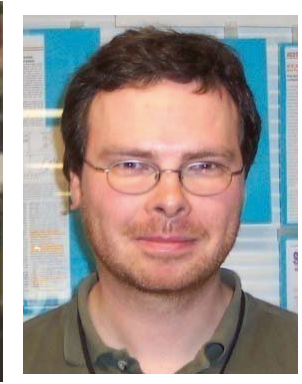

T. Koschny

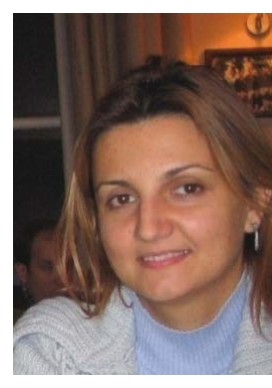

R. Penciu

\section{S. Foteinopoulou}

D. Guney

J. Zhou

B. Wang

\section{Thank you!}

M. Wegener's group at Karlsruhe

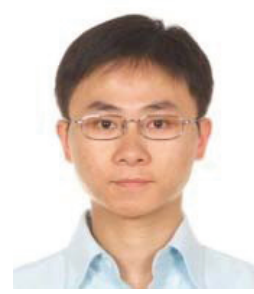

N.H. Shen

\section{Relevant publications}

-R. S. Penciu, M. Kafesaki, Th. Koschny, E. N. Economou, and C. M. Soukoulis, Magnetic response of nanoscale left-handed metamaterials, Phys. Rev. B (2010).

-D. Guney, Th. Koschny, M. Kafesaki, and C. M. Soukoulis, Connected bulk negative index photonic metamaterials, Opt. Lett. 34, 506 (2009).

•N. H. Shen, S. Foteinopoulou, M. Kafesaki, Th. Koschny, E. Ozbay, E. N. Economou, and C. M. Soukoulis, Compact planar far-field superlens based on anisotropic left-handed metamaterials, Phys. Rev. B 80, 115123 (2009). •J. Zhou, J. Dong, B. Wang, Th. Koschny, M. Kafesaki, and C. M. Soukoulis, Negative refractive index due to chirality, Phys. Rev. B 79, 121104(R) (2009). 